\title{
Biophotonics. Fluorescence and Reflectance in Living Organisms
}

\author{
M. Gabriela Lagorio, ${ }^{1,2,{ }^{* *}}$ Gabriela B. Cordon, $3,4,{ }^{*}$ Analia Iriel, 5, ${ }^{*}$ Juan M. Romero, ${ }^{1,2,{ }^{*}}$ \\ Julián Faivovich, 6,7,* and Carlos Taboada ${ }^{8, *}$
}

${ }^{1}$ CONICET, Universidad de Buenos Aires, INQUIMAE, Facultad de Ciencias Exactas y Naturales, Buenos Aires, Argentina.

${ }^{2}$ Universidad de Buenos Aires, Facultad de Ciencias Exactas y Naturales, Dpto. de Química Inorgánica, Analítica y Química Física, Ciudad Universitaria. Plaza Gregorio Klimovsky, Pabellón II, 1er piso, C1428EHA, Buenos Aires, Argentina.

${ }^{3}$ Universidad de Buenos Aires, Facultad de Agronomía, Área de Educación Agropecuaria, Buenos Aires, Argentina.

${ }^{4}$ CONICET, Universidad de Buenos Aires, Instituto de Investigaciones Fisiológicas y Ecológicas Vinculadas a la Agricultura (IFEVA), Buenos Aires, Argentina.

${ }^{5}$ CONICET, Universidad de Buenos Aires, Instituto de Investigaciones en Producción Animal (INPA), Facultad de Ciencias Veterinarias, Buenos Aires, Argentina.

${ }^{6}$ División Herpetología, Museo Argentino de Ciencias Naturales "Bernardino Rivadavia", Consejo Nacional de Investigaciones Científicas y Técnicas (CONICET), Ciudad de Buenos Aires C1405DJR, Argentina.

${ }^{7}$ Departamento de Biodiversidad y Biología Experimental, Facultad de Ciencias Exactas y Naturales, Universidad de Buenos Aires, Ciudad de Buenos Aires C1428EHA, Argentina.

${ }^{8}$ Department of Biology, Duke University, Durham, NC, 27708, USA

* These authors contributed equally to this work.

** Corresponding author. E-mail: mgl@qi.fcen.uba.ar

\begin{abstract}
The light that emerges from a biological entity is relevant from many aspects. In the first place, it allows the construction of the organism's image and consequently it is responsible for visual perception and communication. Secondly, it can become an important tool in obtaining both physiological and chemical information from the observed entity, in a non-destructive way. When an organism is illuminated, the non-absorbed energy emerges as transmitted or reflected light. Additionally, fluorescence, phosphorescence or bioluminescence may be emitted. In our research group, we have studied and modelled the light released as reflectance and fluorescence for different biological systems like flowers, fruits, plant leaves,

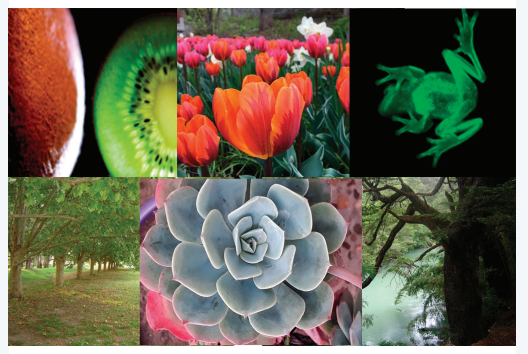
canopies, bird's plumage and amphibians. In this review, we present the advances we have made in this area. They range from the development of theoretical approaches to the implementation of optical methodologies for practical applications. The analysis of light interaction with biological material, which is the domain of biophotonics, has recently acquired great importance in view of the increasing use of optical techniques to the study of living tissues. However, the interpretation of the photophysical and spectroscopic properties of these systems is usually complicated by several factors: elevated chromophore's concentration, optical inhomogeneity, multi-scattering of photons and presence of multi-layered structures in most cases. Because of these, the accurate modelling of the interaction with light helps to avoid artifacts and to better interpret the processes that take place. Physical models used in the analysis of chlorophyll fluorescence in leaves and canopies with application in remote sensing, optical methodologies for food control and quantification of fluorescence in vivo for evaluation of its biological relevance are examples of the use of the emission of light and will be presented in this review.
\end{abstract}

\section{Keywords:}

chlorophyll fluorescence, fluorescence quantum yield, scattering media, light re-absorption, fluorescence modelling, plant biophotonics, optical biosignals 


\section{Introduction}

The study of light and its interaction with matter has been relevant since ancient times and has now acquired an unusual prominence within the field of biophotonics [1].

The term biophotonics comes from the Greek "bios= life" and "phos= light" and the word photonics involves all methodologies and technologies that generate, manipulate and detect photons (UV, visible or IR). So, biophotonics can be briefly defined as the study of optical processes in biological systems. It is concerned with the development of spectroscopic and imaging techniques to non-destructively obtain information from intact cells, organelles, tissues or organisms. In practice, it is a real challenge because biological tissues are usually rich in chromophores, optically non-homogeneous, multi-layered and multi-dispersive in photons. These features of biological entities complicate the interpretation of the light processes that take place in them and physical models to achieve a thorough comprehension of what is really happening in the system are necessary. Modelling also allows for the correction of distortions and artifacts and therefore introduces greater reliability in the information obtained from the analysis of the light released by the material [2].

Biophotonics is applicable to any type of biological tissue and is particularly useful in biomedical and clinical research activities [3]. Similarly, the field of plant biophotonics has been growing rapidly in recent years [4]. In fact, the optical technology resulting from these studies can improve agricultural production and crop quality. It serves to predict the optimal amount of fertilizers to be used, avoiding overdoses and thus preventing undue environmental damage. It also allows the determination of the harvest time and even the surveillance of vegetables and fruits during the post-harvest period. It is also applicable in environmental sciences since natural or anthropogenic alterations in the surroundings affect organisms and usually induce changes in their absorption, reflectance or emission of light. This is particularly true for plants and algae where there is a great interconnection among light absorption, reflectance, fluorescence and photosynthesis [5].

The study of the light emanating from a biological entity is not only interesting for the aspects described above but also for its primary function as a signal in visual connection and communication. A light stimulus from a "sender" is considered an optical communication signal if it is capable of influencing the behaviour of a "receiver". From a physical point of view, to evaluate the relevance of released photons as visual signals, the characteristics of the emitted light (intensity and wavelength) and the sensitivity spectral curves of the optical photoreceptors of the receiver should be analysed in conjunction [6].

This review addresses the analysis of light emanating from biological entities, with emphasis on reflected light and light emitted as fluorescence. Various physical models are presented to correct distortions and to improve the understanding of what is actually observed in the different systems. Advances in the interpretation of signals in the field of biology, recent contributions in the area of chlorophyll fluorescence to assess plant health and the development of non-destructive optical methods for food quality assessment are shown.

\section{Reflectance in biological systems}

\subsection{Connecting reflectance with chromophore concentration}

When a beam of light strikes a tissue, some of the light is absorbed, some is transmitted, and some is reflected. In turn, some of the absorbed light can be emitted as luminescence.

In a general case, reflected light consists of two components: specular and diffuse reflection. While the specular component is reflected at one definite angle, diffuse reflection is scattered in all directions. In fact, the diffuse component is due to the penetration of a fraction of the incoming light into the sample and a subsequent return of the non-absorbed radiation to the surface after multiple scattering at the boundaries present inside the material [7]. In biological entities the diffuse component and consequently light scattering are usually remarkably large.

One of the most used theories to quantify the interaction of light with materials containing small particles which absorb and scatter radiant energy is the Kubelka-Munk (K-M) approach. This theory applies to diffuse reflection and it assumes that the sample is made up of randomly distributed uniformly absorbing fragments. It is a two-flow theory (only two flows: a flow in the direction of incident light and a flow in the direction of reflected light are assumed to move in opposite directions perpendicular to the irradiated surface) [7]. 
For an infinitely thick layer with zero transmittance, K-M theory predicts that the remission function $(\boldsymbol{F}(\boldsymbol{R}))$, which can be calculated by measuring the reflectance $\left(\boldsymbol{R}_{\infty}\right)$ of the thick opaque layer, is proportional to the chromophore concentration in the sample (eq. 1)

$F(R)=\frac{\left(1-R_{\infty}\right)^{2}}{2 R_{\infty}}=\frac{k}{s}=\frac{\sum_{i} w_{i} k_{i}}{\sum_{i} w_{i} s_{i}}$

In the above equation, $\boldsymbol{k}$ and $\boldsymbol{s}$ are the absorption and scattering coefficients, respectively, with $k=2 \varepsilon C$ and $s=2 \sigma$, where $\varepsilon$ is the molar Napierian absorption coefficient, and $C$ the chromophore concentration expressed in mol per unit volume and $\sigma$ is the fraction of light scattered per unit path length in the sample.

When a mixture of chromophores is present, global absorption and dispersion coefficients may be considered additive functions of the respective coefficients $\left(\boldsymbol{k}_{\boldsymbol{i}}\right.$ and $\left.\boldsymbol{s}_{\boldsymbol{i}}\right)$ for the components, weighed according to their mass fractions ( $\left.\boldsymbol{w}_{\boldsymbol{i}}\right)$ (see the fourth member in eq. 1).

Provided that diffuse reflectance, $\boldsymbol{R}$, and diffuse transmittance, $\boldsymbol{T}$, for a single transmitting layer of the studied material is known, separate values for $\boldsymbol{k}$ and $\boldsymbol{s}$ coefficients may be calculated from eqs. 2 to 6 [8].

$$
\begin{aligned}
& k=\frac{a-1}{a+1} \log b \\
& s=\frac{2 a}{a^{2}-1} \log b \\
& a=\frac{1+R^{2}-T^{2}+\Delta}{2 R} \\
& b=\frac{1+T^{2}-R^{2}+\Delta}{2 T}
\end{aligned}
$$

$\Delta^{2}=(1+R+T)(1+R-T)(1+T-R)(1-R-T)$

Both $\boldsymbol{F}(\boldsymbol{R})$ and $\boldsymbol{k}$ are very valuable parameters when information about the content of a certain component in a lightscattering material is required. Several examples in biological samples can be found in literature. For instance, a linear relation was found for $\boldsymbol{F}(\boldsymbol{R})$ values at $533 \mathrm{~nm}$ in Rhododendron indicum petals as a function of anthocyanin chemically determined concentration (Figure 1) [9]. Good linear correlations were also obtained for plant leaves between the water content in $\mathrm{mmol} / \mathrm{cm}^{2}$ and $\mathrm{F}(\mathrm{R})$ at $1456 \mathrm{~nm}$ as for total chlorophyll in $\mathrm{mmol} / \mathrm{cm}^{2}$ and $\boldsymbol{F}(\boldsymbol{R})$ at $700 \mathrm{~nm}$. The correlation with chlorophyll content was improved when using $\boldsymbol{k}$ at $700 \mathrm{~nm}$ instead of the remission function [10]. This last result is to be expected since $\boldsymbol{F}(\boldsymbol{R})$ is directly proportional to the chromophore concentration but only at constant $\boldsymbol{s}$. In contrast, the proportionality between $\boldsymbol{k}$ and $\boldsymbol{C}$ is kept independently of the value of the $\boldsymbol{s}$ coefficient. 


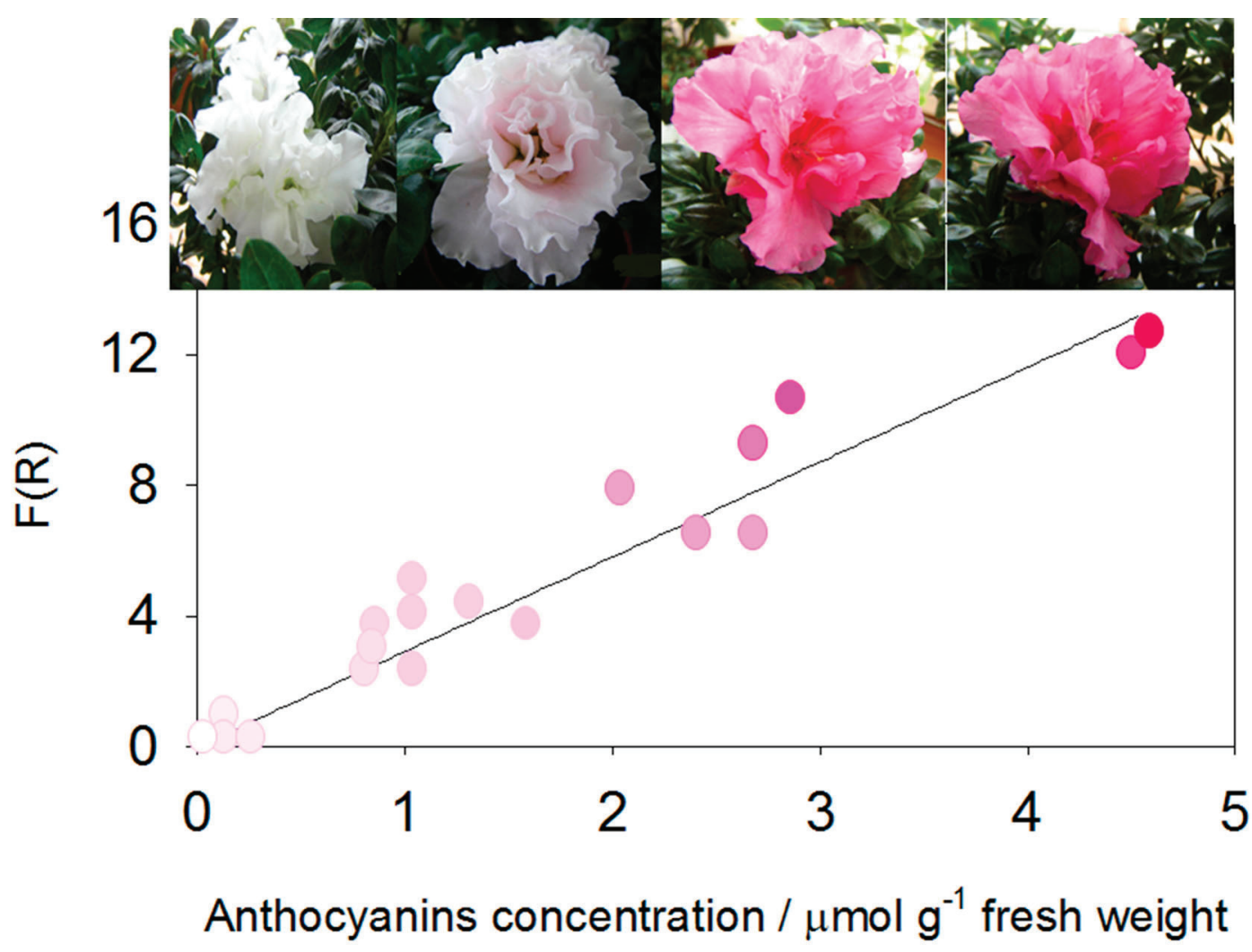

Figure 1. Remission function values at $533 \mathrm{~nm}$ from Rhododendron indicum petals as a function of anthocyanin concentration, $\left(\mathrm{R}^{2}=0.9730\right)$. Reproduced from [9] with permission from the European Society for Photobiology, the European Photochemistry Association, and The Royal Society of Chemistry.

\subsection{Reflectance and colour coordinates. Related but quite different concepts}

Since a relationship between the reflected light and the chromophore concentration can be found, as illustrated above, and since the reflected light is in turn related to the colour coordinates, it is appealing to examine whether it is also feasible to correlate the chromophore concentration with the sample colour.

At this point, it is important to emphasize that the reflectance spectrum of a sample is a property of the material whereas the color is not. Indeed, colour is a sensation created by the brain of an observer. This perception depends not only on the reflectance spectrum of the "sender" but also on the spectral distribution of the light source and the sensitivity curve of the observer's visual photoreceptors.

To transform reflectance spectra to colour coordinates for a particular observer under specific lighting conditions, different color spaces may be used [11]. Cordon and Lagorio used color coordinates to sense various photosynthetic pigments in plant leaves, with spatial resolution, from digital images [12]. First, they obtained correlations between $\boldsymbol{R} \boldsymbol{G B}$ colour coordinates of plant leaves and the pigment concentration determined by a standard wet method. Then, these authors obtained images of green and senescent leaves with a commercial scanner (TIFF format with a resolution of 300 dpi and a depth of 24 bits). Using the program ERDAS IMAGINE 8.4, they converted the images from TIFF to IMG format and then separated into their $\boldsymbol{R}, \boldsymbol{G}$ and $\boldsymbol{B}$ bands. Finally, using the correlations between each color coordinate and the pigment concentration, maps of pigment contents were obtained in gray scale. To improve visual interpretation, the grayscale images were transformed to pseudocolour coding. Figure 2 illustrates the concentration maps obtained from images of Liquidambar styraciflua leaves. 


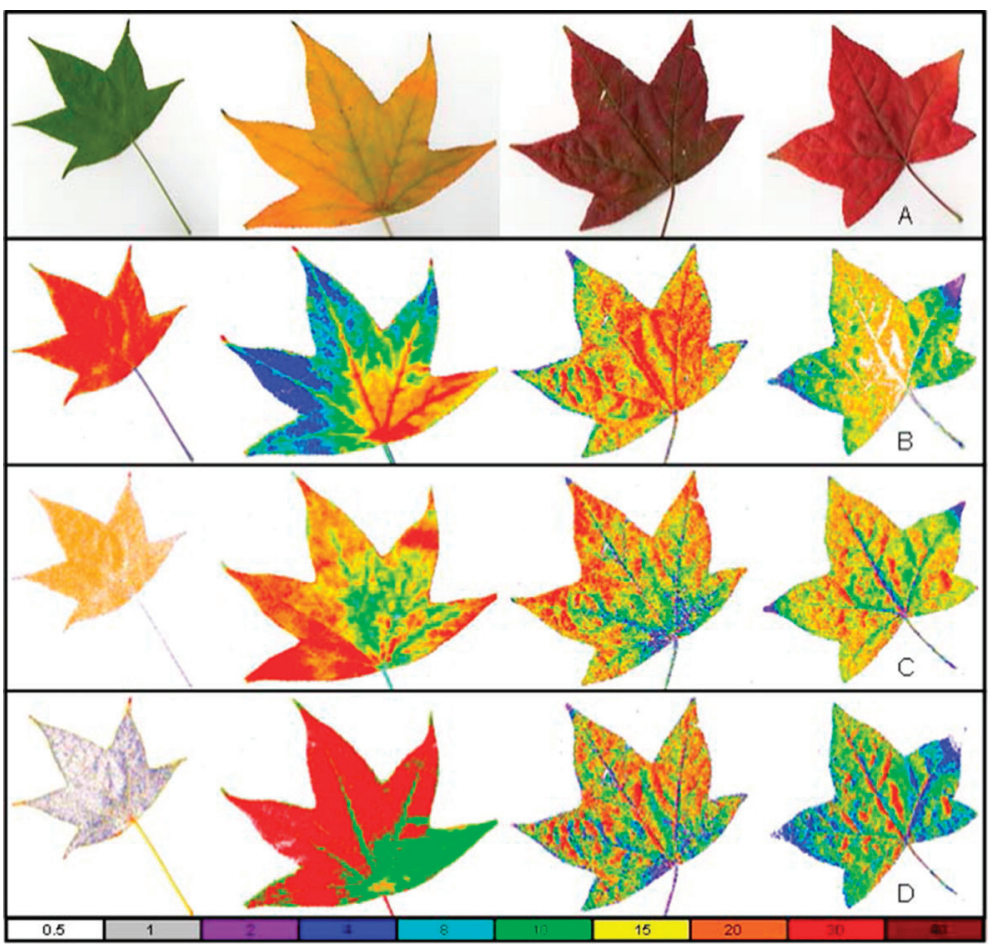

Figure 2. A. Images of Liquidambar styraciflua captured with a commercial scanner. B, C and D. Concentration maps of total chlorophyll, carotenoids and anthocyanins, respectively. Numbers express concentration in $\mathrm{nmol} / \mathrm{cm}^{2}$.

\subsection{Reflectance as a bio-signal. Quantum catches and vision contrasts}

Some examples to illustrate the usefulness of reflectance and imaging as tools for determining analyte contents were exemplified in the previous sections. In nature, however, reflected light provides photons detected in visual perception. Thus, reflected light plays a primary role in optical biosignaling and in visual communication.

In this context, an important parameter is the quantum catch or stimulus strength, $\boldsymbol{Q}_{i}$, which represents the relative amount of light absorbed by a given photoreceptor per unit time (eqs. 7-9) [6].

$Q_{i=} k_{i} P_{i}$

With $P_{i}=\int_{300}^{700} R(\lambda) \cdot S_{i}(\lambda) \cdot I(\lambda) \cdot d \lambda$

$k_{i}=1 /\left(\int_{300}^{700} S_{i}(\lambda) I(\lambda) d \lambda\right)$

Where $\boldsymbol{i}$ stands for the type of the photoreceptor (L: long wavelength, M: medium wavelength, S: short wavelength or VS: very short wavelength), $\lambda$ is the wavelength, $S_{i}(\lambda)$ is the photoreceptor spectral sensitivity, $R(\lambda)$ is the reflectance spectrum of the observed object, $I(\lambda)$ is the illuminant spectrum and $d \lambda$ is the differential of wavelength.

Even more important to distinguish an object from its background is the chromatic contrast $\left(\boldsymbol{C}_{i}\right)$ which may be calculated dividing $\boldsymbol{P}_{i}$ by the total number of photons absorbed by receptor $\boldsymbol{i}$ from the background $\left(\boldsymbol{P}_{i b}\right)$ (eqs. 10 and 11$)$.

$C_{i}=P_{i} / P_{i b}$

$P_{i b}=\int_{300}^{700} R_{b}(\lambda) \cdot S_{i}(\lambda) \cdot I(\lambda) \cdot d \lambda$

Figure 3 shows typical values for quantum catches and contrasts calculated for the visual perception of bees, birds and humans when looking at Rhododendron indicum petals with different colour and illuminated with a standard D65 light simulating daylight. 

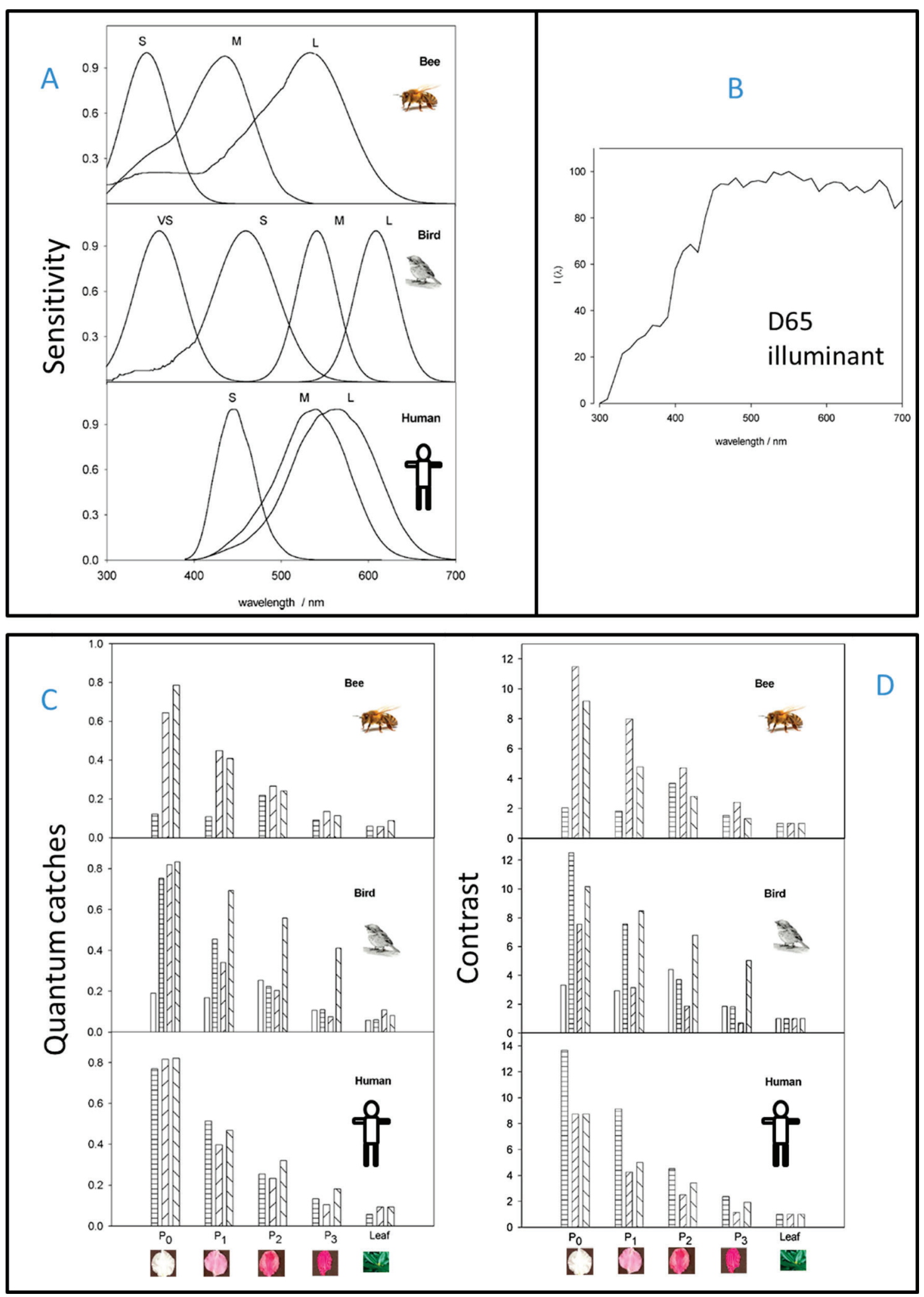

Figure 3. A) Photoreceptor sensitivities for the honeybee Apis mellifera, for the bird Leiothrix lutea and for humans. The letters VS, S, M and L mean very short, short, medium and long wavelengths, respectively. B) Spectral distribution of D65 illuminant. C) Quantum catches and D) Contrasts to background for the honeybee Apis mellifera, for the bird Leiothrix

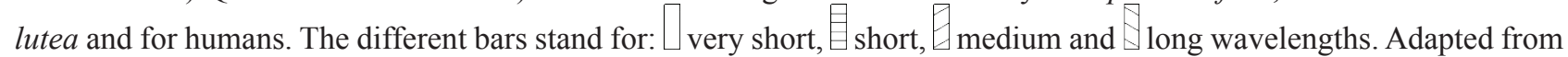
Ref. [6] with permission from the European Society for Photobiology, the European Photochemistry Association, and The Royal Society of Chemistry. 
Humans see the white petal as achromatic because their three photoreceptors are excited in a similar degree during the observation in daylight (see Figure 3C). In contrast, a bird or bee looking at the same flower does not capture the same number of photons in each of their receptors so that the "white" petals will look chromatic to their eyes. Figure 3A shows that the darker a tissue is, the lower the value of the quantum catches, which is an obvious consequence of the lower number of photons reaching the eye from the light reflected by the petal. It is also evident that bees do not have photoreceptors in the red and then, they can poorly capture light with long visible wavelengths.

It is assumed that the contrast between the flowers and the background helps pollinators to find flowers. The greatest contrast to the background in Figure 3D is given for the white flowers for all the observers. In the case of dark pink flowers, the contrast to the background is low for humans and bees. For birds, on the contrary, the contrast of the red receptor is relatively high. This is consistent with the fact that red and pink flowers are attractive to birds and are mostly pollinated by them [ 6 and references therein]. Therefore, as a general rule, in order to evaluate visual signals, it is essential to handle the concepts of quantum catches and contrasts, and if feasible, to complement this information with behavioural experiments.

From this, the enormous relevance of having experimental information on the sensitivity curves of the optical photoreceptors of different species becomes evident. Although for humans and a few other species these aspects have been studied in depth, this is not the case for most animals. This is certainly an interesting field to develop in the field of photobiology.

\section{Fluorescence in biological systems}

\subsection{How to quantify fluorescence in scattering media. Determination of fluorescence quantum yield}

To analyse the relevance of fluorescence as a signal in nature, it is essential to quantify fluorescence. In the case where the number of fluorescence photons is negligible compared to that of reflected photons, the fluorescence has little chance of having biological relevance. On the contrary, if the number of emitted photons is comparable with the number of reflected photons, then a contribution of the fluorescence to the image of the organism will surely take place. These evaluations should be conveniently complemented with behavioural experiments to fully discuss the possible role of fluorescence in communication.

Knowledge of the fluorescence quantum yield (number of photons emitted per absorbed photon) is an initial step for the quantification of fluorescence. The intact organisms generally have considerable scattering and high chromophore concentration and consequently the quantification of fluorescence cannot be carried out by traditional methods in solution. Several methodologies are reported in literature for the determination of fluorescence quantum yields in scattering media. Some of them use an integrating sphere [13-17], whereas others use a standard spectrofluorometer without sphere [17]. In our studies on intact biological samples [6, 18-20] we proceeded according to reference [17] for the determination of the fluorescence quantum yield.

In brief (see also Figure 4):

1. The sample is placed in front-face geometry within a standard steady-state spectro-fluorometer and the total number of emitted photons $\boldsymbol{J}_{\boldsymbol{f}}$, at excitation wavelength $\boldsymbol{\lambda}_{\boldsymbol{0}}$, is obtained as the area under the emission spectra.

2. The sample is then replaced by a blank $\left(100 \%\right.$ reflecting material as $\left.\mathrm{BaSO}_{4}\right)$ and an optical filter (for instance a glass Schott, NG4, $0.5 \mathrm{~mm}$ ) is inserted at the emission slit to avoid detector damage. The light reaching the detector is registered by varying the emission wavelength from $\left(\lambda_{0}-15 \mathrm{~nm}\right)$ to $\left(\lambda_{0}+15 \mathrm{~nm}\right)$ and the number of photons scattered by the blank $\left(\boldsymbol{J}_{0}\right)$ are calculated from the area under the recorded peak divided by the transmittance spectrum of the filter.

3. Step 2 is repeated replacing the blank by the sample and the integrated reflected light from the sample, $\boldsymbol{J}$, is obtained.

To take into account differences in light scattering between sample and blank, two additional measurements are performed:

4. Keeping the filter at the emission window, the sample is excited at a wavelength where the sample does not absorb and emission spectra is recorded from $(\lambda-15 \mathrm{~nm})$ to $(\lambda+15 \mathrm{~nm})$. Under these conditions, the area under the recorded peak divided by the transmittance spectrum of the filter yields the integrated scattered light intensity from the sample $(\boldsymbol{I})$. 
5. Proceeding as in step 4 with the blank, the integrated scattered light intensity from the blank $\left(\boldsymbol{I}_{\boldsymbol{0}}\right)$ is obtained.

Then, the fluorescence quantum yield is calculated as:

$\Phi_{f}=\frac{\text { number of emitted photons }}{\text { number of absorbed photons }}=\frac{J_{f}}{J_{0}\left(\frac{I}{I_{0}}\right)-J}$

In the case that the absorption of the sample at wavelength $\lambda$ is low but not strictly null (reflectance, $R \neq 1$ ), the correction factor in eq. 12 should be replaced by $\boldsymbol{I} /\left(\boldsymbol{I}_{0^{*}} \boldsymbol{R}\right)$ (For more details see ref. [20]). Following this methodology, we quantified and reported sexual dichromatism in fluorescence in avians for the first time [19]. In fact, sexual dichromatism in birds, which is very frequent, is the presence of sexual differences in plumage coloration. Sometimes, these differences cannot be detected by humans but are discernible to avian eyes. Furthermore, when plumage emits, there may be differences also in fluorescence between sexes. For the blue-winged parrotlet Forpus xanthopterygius, the male rump emits blue fluorescence that is absent in the female. Additionally, the male chest emits a green fluorescence which is much more intense than that emitted by females.

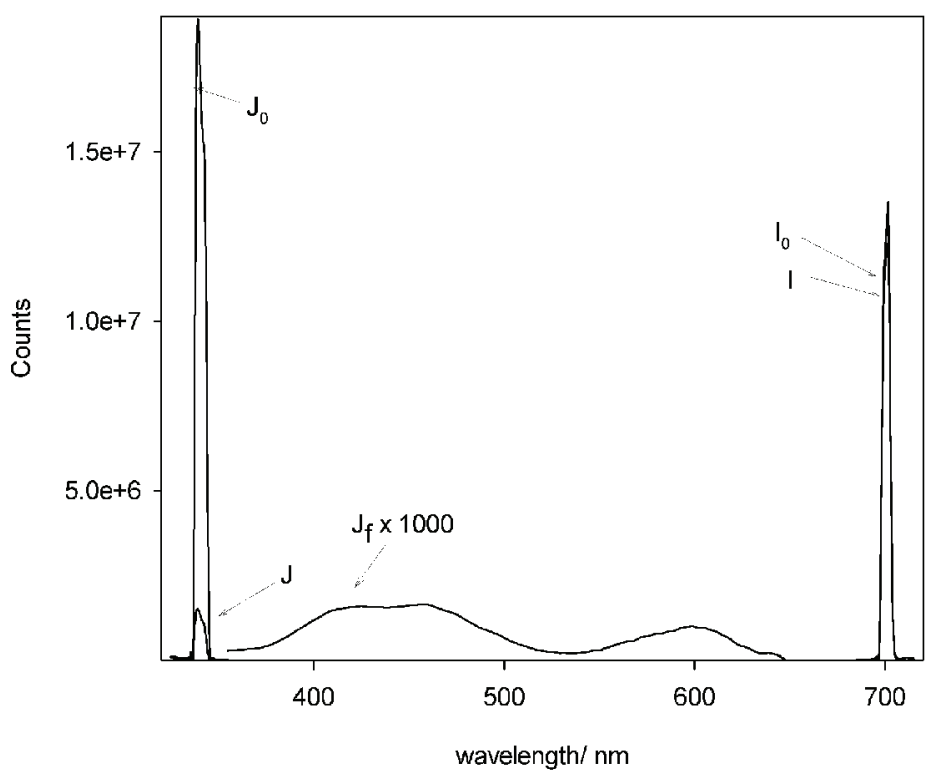

Figure 4. Raw data necessary for the determination of the fluorescence quantum yield of light scattering samples. $\boldsymbol{J}_{\boldsymbol{0}}$ : integrated scattered light from the blank, $\boldsymbol{J}$ : integrated scattered light from the sample, $\boldsymbol{J}_{\boldsymbol{f}}$ : emission spectrum from the sample, I: scattered light from the sample, $\boldsymbol{I}_{\mathbf{0}}$ : scattered light from the blank. Reproduced from [6] with permission from the European Society for Photobiology, the European Photochemistry Association, and The Royal Society of Chemistry.

Determinations of fluorescence quantum yields in scattering media are reviewed in [16]

\subsection{Fluorescence vs. Reflectance in nature. How to quantitatively estimate the rela- tive importance of fluorescence}

From a biological perspective, an important question is whether fluorescence in organisms plays a role or is simply a nonfunctional consequence of the chemical nature of the molecules involved. The search for the answer to this question can be addressed from diverse aspects and by both behavioural and photophysical experiments.

Focusing on optics, a first idea of the contribution of fluorescence can be obtained by comparing the number of fluorescent photons with the number of reflected ones. For analysing the relevance of fluorescence in flowers [6, 18], as a first step, we have compared the fluorescence intensity per incident photon, calculated as $\phi_{f} \cdot f_{a}(\lambda)$ (where $f_{a}(\lambda)$ is the fraction of photons absorbed by the sample at wavelength $\lambda$ ) with the fraction of reflected photons. For the opaque samples, as no light was transmitted through the material, $\boldsymbol{f}_{a}(\lambda)$ was directly obtained as $[\mathbf{1 - R}(\lambda)]$. Nevertheless, a more rigorous analysis that properly includes the environmental lighting conditions is even more convenient for a better comprehension of the system and is described below. 
When a fluorescent organism is illuminated by a polychromatic light, with a spectral distribution $I(\lambda)$, the total number of fluorescence photons may be calculated by eq. 13 .

$$
F_{t}=\int_{\lambda_{\text {ex }}}^{\lambda_{e x 2}} \phi_{f}(\lambda) \cdot I(\lambda) \cdot f_{a}(\lambda)
$$

where integration is performed over the interval of excitation wavelengths $\lambda_{e x 1}-\lambda_{e x 2}$.

For a translucent sample, $f_{a}(\lambda)$ is calculated as $[1-R(\lambda)-T(\lambda)]$ which reduces to $[1-R(\lambda)]$, when transmittance $(T)$ is zero.

The spectral photon flux distribution of fluorescence $\boldsymbol{F}_{t}(\lambda)$ may be calculated as the product between $\boldsymbol{F}_{t}$ and the normalized spectral distribution of fluorescence $g(\lambda)$ (with $\int g(\lambda)=1$ ):

$F_{t}(\boldsymbol{\lambda})=F_{t} g(\boldsymbol{\lambda})$

Similarly, the photon flux distribution of reflected photons is estimated by equation (15).

$R_{t}(\boldsymbol{\lambda})=R(\boldsymbol{\lambda}) \cdot I(\boldsymbol{\lambda})$

Finally, for a given spectral range $\lambda_{1}-\lambda_{2}$, the contribution of fluorescence $\left(F_{\text {cont }}\right)$ to the total number of photons released by the entity, may be estimated as the fraction of fluorescence photons relative to the total number of photons (reflected plus fluorescent) according to eq. 16 .

$$
F_{\text {cont }}=\frac{\int_{\lambda_{1}}^{\lambda_{2}} F_{t}(\lambda) d \lambda}{\int_{\lambda_{1}}^{\lambda_{2}}\left(F_{t}(\lambda)+R_{t}(\lambda)\right) d \lambda}
$$

This approach was applied to estimate the relevance of fluorescence for the first naturally fluorescent frog reported in scientific literature [20]. In that case, fluorescence contribution turned out to be almost $30 \%$ under twilight conditions (Figure 5), a result very interesting as it suggests a possible role of fluorescence in visual communication among individuals [21].
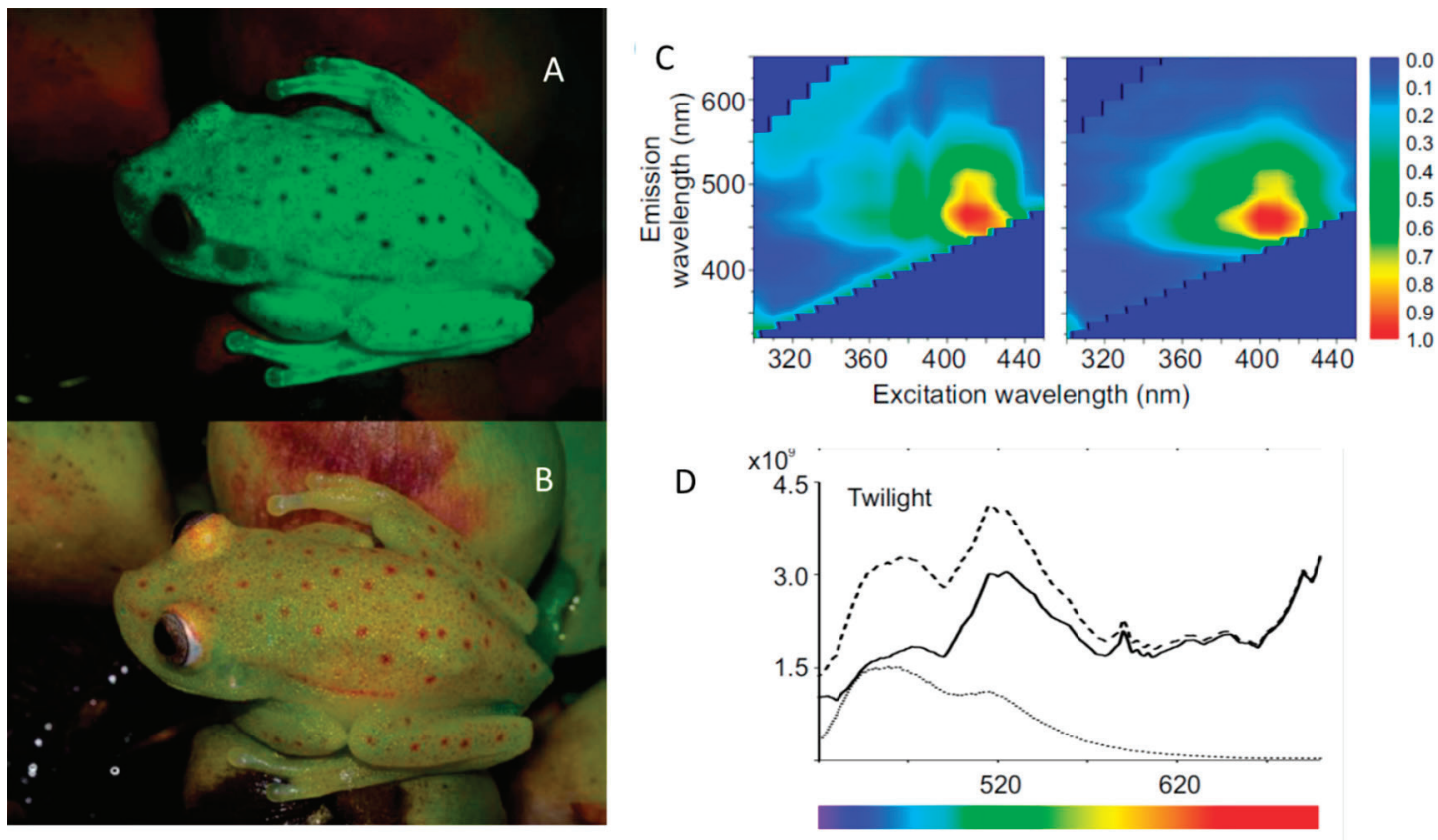

Wavelength $(\mathrm{nm})$

Figure 5. Fluorescence in the frog Boana punctata. Adult male (A) under UV-blue light and (B) white light. Excitationemission matrices of the dorsal surfaces of female (Left) and male (Right) specimens (C). Contribution of fluorescence to total emerging light. (B) Spectral photon flux (photons $/\left[\mathrm{cm}^{2} . \mathrm{s} . \mathrm{nm}\right]$ ) emerging from the dorsal surfaces of one of the 
specimens under twilight illumination. Reflected light (solid line) fluorescence light (dotted line), and the sum of both components (dashed line). Adapted from [20] (Taboada, C.; Brunetti, A. E.; Pedron,F. N.; Carnevale Neto, F.; Estrin, D. A.; Bari, S. E.; Chemes, L. B.; Peporine Lopes, N.; Lagorio, M. G.; Faivovich, J. Naturally occurring fluorescence in frogs. PNAS, 2017, 114, 3672-3677) with permission from the National Academy of Sciences, USA.

\subsection{Fluorescence in multilayered systems. How to retrieve the observed total fluo- rescence from the spectroscopic information of each layer}

It is common to find multilayered structures in biological organisms. Very often, each part fluoresces in a specific way and contributes to the total fluorescence emitted by the intact organism. In these cases, it may be interesting to know where the global fluorescence mainly originates. Modelling can help to provide an answer and determine the contribution of each individual layer.

Let's consider a system of $\boldsymbol{n}$ layers each characterized by their respective reflectance $\boldsymbol{R}_{\boldsymbol{i}}$, transmittance $\boldsymbol{T}_{\boldsymbol{i}}$ and fluorescence intensity $\boldsymbol{I}_{f i}$ (see Figure 6). As the excitation light $\left(\boldsymbol{I}_{0}\right)$ enters the system, it suffers attenuation due to the filter effect of each layer. It can be roughly considered that a photon flux $\boldsymbol{I}_{0}$ reaches layer 1 , a flux $\boldsymbol{I}_{0} \cdot \boldsymbol{T}_{1}\left(\boldsymbol{\lambda}_{0}\right)$ (where $\lambda_{0}$ is the excitation wavelength) reaches layer $2, I_{0} \cdot T_{1}\left(\lambda_{0}\right) \cdot T_{2}\left(\lambda_{0}\right)$ reaches layer 3 and so on. In each layer, the generated flux of fluorescent photons is the product of the fluorescence quantum yield $\left(\boldsymbol{\phi}_{f}\right)$, the photon flux reaching the layer $\left(\boldsymbol{I}\left(\boldsymbol{\lambda}_{0}\right)\right)$ and the fraction of light absorbed by the sample $\left(f_{a i}\right)$ (equation 17)

$I_{f i}=\phi_{f i} . I\left(\lambda_{0}\right) \cdot f_{a i}\left(\lambda_{0}\right)=\phi_{f i} \cdot I\left(\lambda_{0}\right) \cdot\left(1-R_{i}\left(\lambda_{0}\right)-T_{i}\left(\lambda_{0}\right)\right)$

The fluorescence originated in each layer travels outwards and it is attenuated by the transmittance of the upper layers. The global fluorescence may be estimated as the sum of all the contributions (equation 18).

$I_{f t}=I_{f 1}(\lambda)+I_{f 2}(\lambda) \cdot T_{1}(\lambda)+I_{f 3}(\lambda) \cdot T_{1}(\lambda) T_{2}(\lambda)+\cdots+I_{f n}(\lambda) \cdot T_{1}(\lambda) T_{2}(\lambda) \ldots T_{n-1}(\lambda)$

From eqs. 17 and 18, eq. 19 arises:

$I_{f t}=\phi_{f 1} \cdot I_{0}\left(\lambda_{0}\right) \cdot\left(1-R_{1}\left(\lambda_{0}\right)-T_{1}\left(\lambda_{0}\right)\right)+\phi_{f 2} \cdot I_{0}\left(\lambda_{0}\right) \cdot\left(1-R_{2}\left(\lambda_{0}\right)-T_{2}\left(\lambda_{0}\right)\right) \cdot T_{1}\left(\lambda_{0}\right) T_{1}(\lambda)+$

$\phi_{f 3} \cdot I_{0}\left(\lambda_{0}\right) \cdot\left(1-R_{3}\left(\lambda_{0}\right)-T_{3}\left(\lambda_{0}\right)\right) \cdot T_{1}\left(\lambda_{0}\right) \cdot T_{2}\left(\lambda_{0}\right) T_{1}(\lambda) T_{2}(\lambda)+\cdots+\phi_{f n} \cdot I_{0}\left(\lambda_{0}\right) \cdot\left(1-R_{n}\left(\lambda_{0}\right)-\right.$

$\left.T_{n}\left(\lambda_{0}\right)\right) \cdot T_{1}\left(\lambda_{0}\right) \cdot T_{2}\left(\lambda_{0}\right) \ldots T_{n-1}\left(\lambda_{0}\right) \cdot T_{1}(\lambda) \cdot T_{2}(\lambda) \ldots T_{n-1}(\lambda)$ $(\lambda)$.

In eq. 19 , it is important to highlight the difference between the excitation wavelength $\left(\lambda_{0}\right)$ and the emission wavelength

It should be also noted that eq. 19 may be re-written in terms of the experimentally determined fluorescence intensities of each layer $\left(\boldsymbol{I}_{f i}\right.$ iso $)$ when they are isolated and irradiated directly by a photon flux $\boldsymbol{I}_{0}$ (eq. 20)

$$
\begin{aligned}
& I_{f t}=I_{f 1}^{\text {iso }}+I_{f 2}^{\text {iso }} \cdot T_{1}\left(\lambda_{0}\right) T_{1}(\lambda)+I_{f 3}^{\text {iso }} \cdot T_{1}\left(\lambda_{0}\right) \cdot T_{2}\left(\lambda_{0}\right) T_{1}(\lambda) T_{2}(\lambda)+\cdots+ \\
& I_{f n}^{\text {iso }} \cdot T_{1}\left(\lambda_{0}\right) \cdot T_{2}\left(\lambda_{0}\right) \ldots T_{n-1}\left(\lambda_{0}\right) \cdot T_{1}(\lambda) \cdot T_{2}(\lambda) \ldots T_{n-1}(\lambda)
\end{aligned}
$$

It should be noticed that if $\boldsymbol{I}_{f}, \boldsymbol{R}$ and $\boldsymbol{T}$ are experimentally determined for each layer, it is possible to validate the model by comparing the experimental global fluorescence spectrum with the calculated spectrum resulting from the sum of contributions given by eq. 20. Moreover, the relative participation of each layer may be estimated. This type of physical modeling is extremely simplified since it does not take into account multiple reflections. Even so, it was successful when applied to the comprehensive study of the global spectrum of the frog Boana punctata [20] and that of the kiwi fruit [22]. In the supplementary material of ref. [22] the validity of neglecting multiple reflections for the excitation beam and for the fluorescence emission (in the case of kiwifruit) is thoughtfully discussed.

This model was also applied for the analysis of fluorescence in eggplants fruits [23]. In this case, the observed spectrum for the whole intact fruit was reproduced by eq. 20 with an additional term which stood for the peel fluorescence generated internally, reflected by the pulp and filtered by the peel. In other words, when an internal layer $(\boldsymbol{I L})$ has high reflectance, as it happens for the eggplant pulp, the internal fluorescence of the upper layer $(\boldsymbol{U} \boldsymbol{L})$ can be reflected in the lower layer $(\boldsymbol{L} \boldsymbol{L})$ and comes out after suffering a filtering effect when it passes through the upper layer. The additional term for the system of two layers may be written then as $\boldsymbol{I}_{f U L} \cdot \boldsymbol{R}_{L L} \cdot \boldsymbol{T}_{U L}$ (details can be found in ref. [23]). 
Using this kind of modelling it was concluded that skin and lymph were the layers mostly contributing to the observed fluorescence in the Boana punctata frog. In the case of kiwi fluorescence, it could be shown that the relative contribution of the pulp fluorescence to the total emission for the intact fruit depended on the peel transmittance, which was, in turn, a function of the excitation wavelength. For an excitation in the blue, the contributions were $81 \%$ and $19 \%$ for the peel and the pulp respectively, whereas for excitation in the orange the contributions changed to 75 and $25 \%$, respectively.

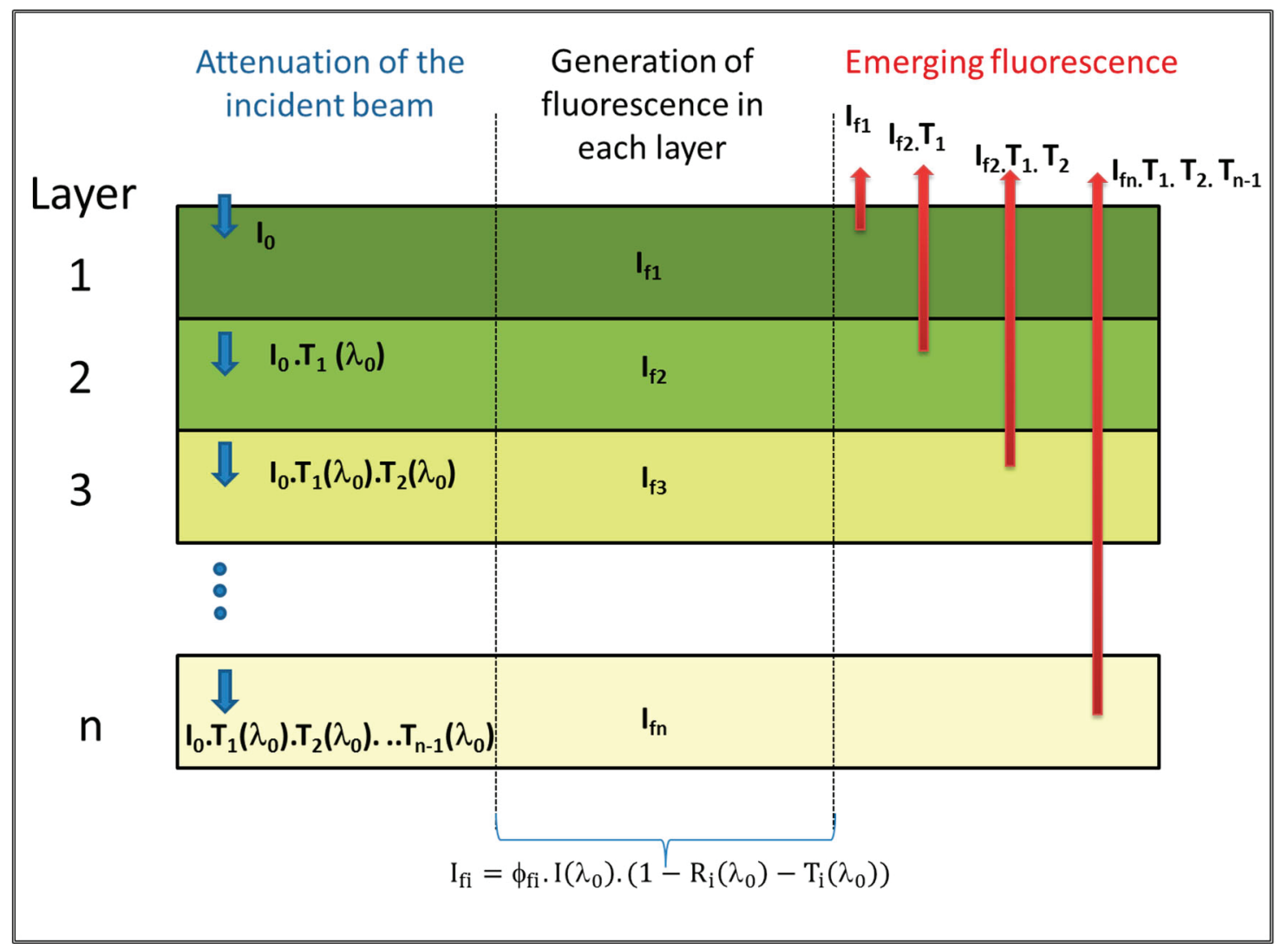

Figure 6. Multilayered system. Pictorial description of: the processes of attenuation of the excitation beam $\left(\boldsymbol{I}_{0}\right)$ through the different layers (left sector), generation of fluorescence in each layer (middle sector) and attenuation of the fluorescence on its way out of the system (right sector).

Future research analyzing how frogs' emission impacts on their chromatic and achromatic contrast with their environment, as seen by predators and by predated species, will be planned. Knowledge on the sensitivity curves of the observer's photoreceptors is essential for that study.

\subsection{Plant biophotonics. Chlorophyll fluorescence: a quite singular type of fluores- cence in nature}

Plants emit fluorescence in the blue and in the red part of the electromagnetic spectrum. Blue fluorescence (around 450 $\mathrm{nm}$ ) is due to emission from phenolic substances (caffeic, ferulic, chlorogenic, coumaric and rosmarinic acids) [24], whereas red emission is originated from chlorophyll-a in photosystem II (PSII) and photosystem I (PSI) [25].

Chlorophyll fluorescence is strongly related to photosynthesis and it is used as a tool to assess plant health. The photosynthetic process in plants causes variability in chlorophyll emission. This variable emission is usually analysed by means of a pulse amplitude modulated (PAM) fluorometer [26]. In fact, when dark-adapted leaves are illuminated by a low photon flow, a constant initial fluorescence $\boldsymbol{F o}$ is recorded (Figure 7). If a saturation light pulse is then applied, the primary electron acceptors in the photosynthetic chain are reduced and are unable to acquire another electron before they have effectively passed the received electron on to the next acceptor. As a consequence, the fluorescence emission increases to a maximum $\boldsymbol{F m}$ and subsequently decays. In a typical experiment, an actinic light is then switched on and the fluorescence reaches a peak and then falls to a steady state $(\boldsymbol{F s})$, in a process called fluorescence quenching. This quenching has two contributions: the photochemical quenching, due to the activation of enzymes in carbon metabolism by 
light, and the non-photochemical quenching on account of the enhancement of heat dissipation. Subsequent applications of saturating light pulses lead to increases in fluorescence (Fm') (Figure 7).

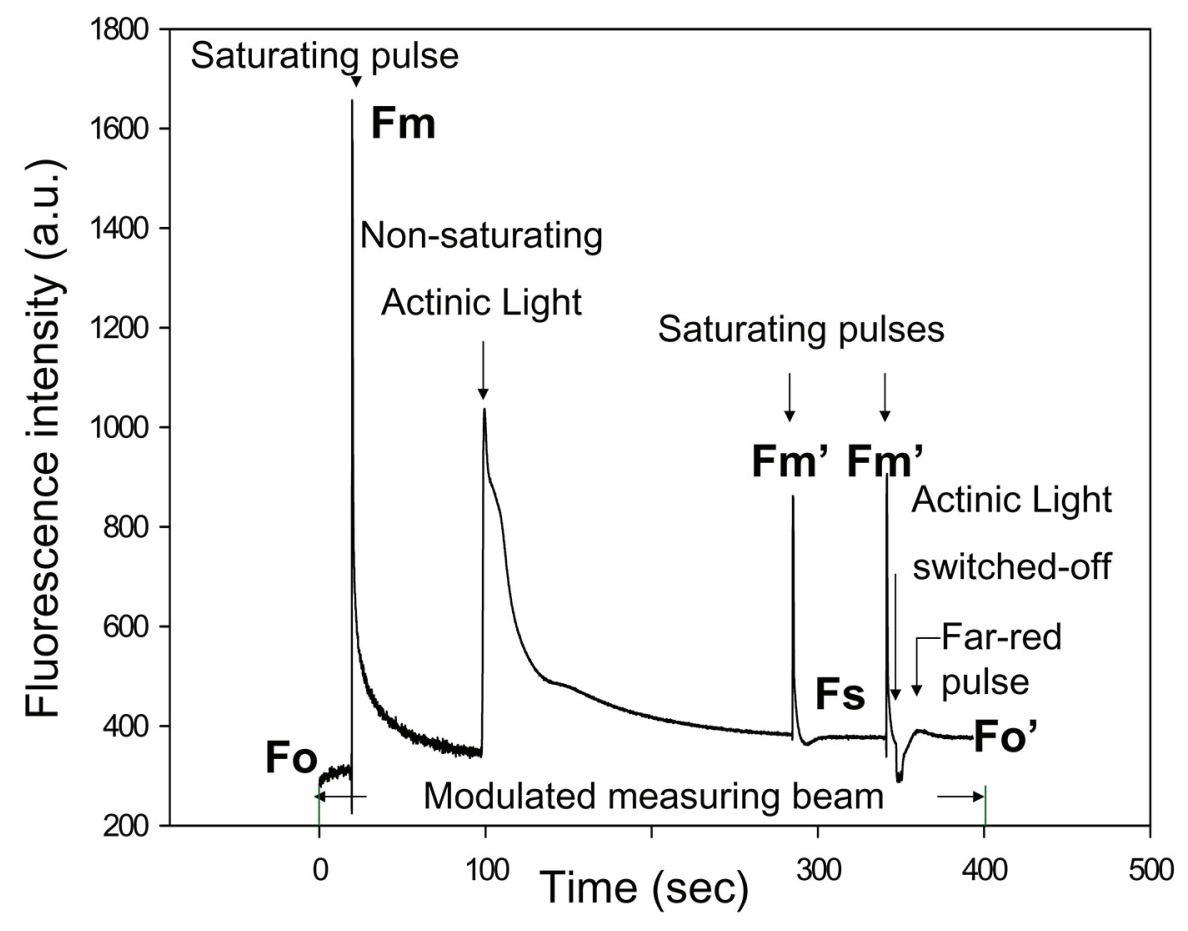

Figure 7. Variable chlorophyll fluorescence recorded with a pulse modulated fluorometer for a typical plant leaf of Spathiphyllum wallisi. Reproduced with permission from Ref. [27] Copyright 2014 Wiley.

Several photosynthetic parameters can be derived from an experiment on variable chlorophyll fluorescence. The main parameters are listed below:

-the maximum quantum yield of photosynthesis for dark-adapted leaves $\boldsymbol{F}_{\boldsymbol{v}} / \boldsymbol{F}_{\boldsymbol{m}}$ (eq. 21 ).

$\frac{F_{v}}{F_{m}}=\frac{F_{m}-F_{0}}{F_{m}}$

-the coefficient for the photochemical quenching, $\boldsymbol{q}_{P}$ (equation (22)).

$q_{P}=\frac{F_{m}^{\prime}-F_{s}}{F_{m}^{\prime}-F_{0}}$

-the coefficient for the non-photochemical quenching, $\boldsymbol{q}_{N P}$ (eq. 23) and its alternative expression $\boldsymbol{N P Q}$ (eq. 24), representing the magnitude of heat dissipation under a given actinic light.

$q_{N P}=\frac{F_{m}-F_{m}}{F_{m}-F_{0}}$

$N P Q=\frac{F_{m}-F_{m}}{F_{m}^{\prime}}$

-the efficiency of PSII, $\boldsymbol{\phi}_{P S I}$ (eq. 25), representing the photosynthetic activity under a defined actinic light.

$\Phi_{P S I I}=\frac{F_{m}^{\prime}-F_{s}}{F_{m}^{\prime}}$ 
- the quantum yield of NPQ, $\phi_{N P Q}$ (eq. 26).

$\Phi_{N P Q}=N P Q \times \frac{F_{s}}{F_{m}}$

- the quantum yield of photophysical decay (representing the deactivation of the excited reaction center at PSII by internal conversion, intersystem crossing and fluorescence, under a particular actinic light), $\boldsymbol{\phi}_{C}$ (eq. 27),

$\Phi_{C}=\frac{F_{s}}{F_{m}}$

The derivation of these relationships and their physical meaning may be found in references [28-32].

It is important to notice that the sum of the last three parameters (each of which is a function of the actinic light intensity) gives unity, so that an increase in one of them leads to a decrease in one or both other two [33]. These parameters enable us to visualize the partition of energy within the plant among photosynthesis, heat dissipation and photophysical decay (which includes fluorescent emission). Clearly, this is of huge value in photosynthesis studies and in evaluating the harnessing of solar energy to produce organic matter.

Under low illumination such as not to induce Kautsky kinetics, a spectral distribution for the initial fluorescence of plant leaves may be recorded. At room temperature, this fluorescence is characterized by two peaks: one in the red, $\boldsymbol{F}_{\text {red }}$ (around $680 \mathrm{~nm}$, due to PSII) and one in the far-red, $\boldsymbol{F}_{\text {far-red }}$ (around $730 \mathrm{~nm}$, due to both photosystems) [27, 34].

The fluorescence ratio $\mathbf{F}_{\text {red }} / \mathbf{F}_{\text {far-red }}$ has been widely related to plant stress and photosystems stoichiometry [23, 33, 35]. However, the spectral distribution of fluorescence at leaf level is distorted compared to the spectral distribution at chloroplast level. This is caused by light re-absorption and greatly affects the red band (Figure 8) [36, 37]. As the connection between the fluorescence ratio and plant physiology is strictly valid when artifacts are absent, it is extremely relevant to correct spectra for light re-absorption processes.

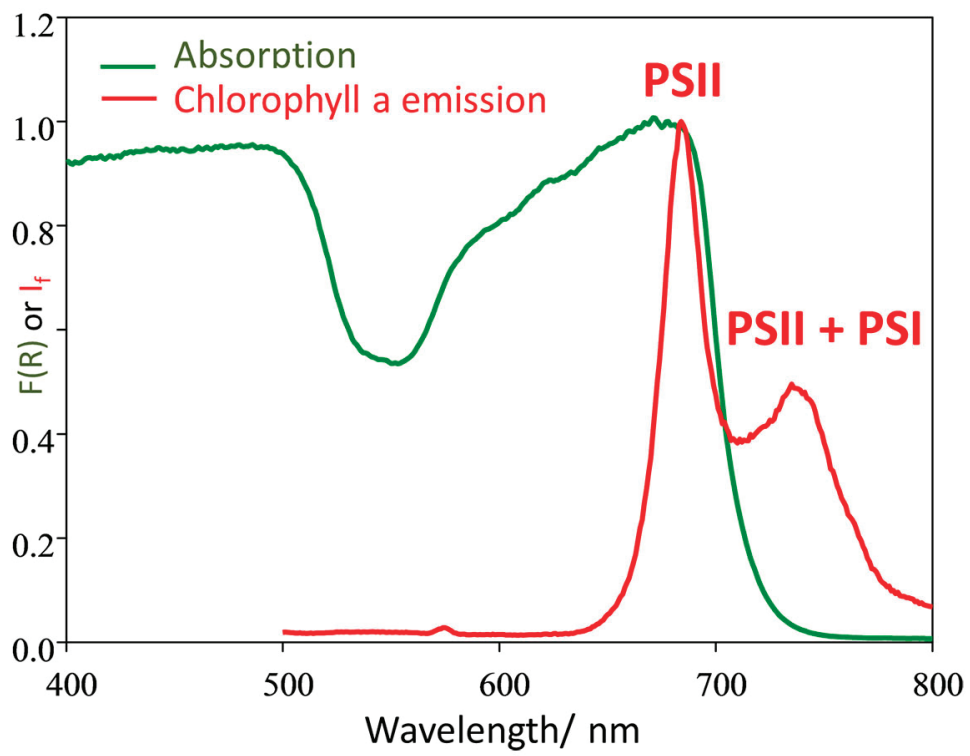

Figure 8. Typical absorption end emission spectra of plant leaves. The red emission band, which is due to PSII emission, significantly overlaps the absorption spectrum and undergoes severe re-absorption processes. The far-red emission band caused by emission from both photosystems is less affected by light re-absorption.

Chlorophyll fluorescence can be measured at different scales: at chloroplast level, at leaf level and at canopy level. In turn, the fluorescence of the canopy can be recorded at ground, airborne or space borne scale. Each level of observation has its own particularities, both in terms of how the experimental measurements are carried out and in developing models capable of correcting distortions by light re-absorption processes. 


\subsubsection{Light re-absorption processes}

\subsubsection{From the leaf to the chloroplast}

In literature, there are three proposed models to correct light re-absorption processes in plant leaves [36, 38-39]. They were compared and discussed in detail in a paper from Cordon and Lagorio [37]. According to the analysis described in this latter paper, one of the correction methods [39] would be subject to conceptual controversies, whereas the other two $[36,38]$ are supported by good validations and lead to similar results.

The main objective of the light re-absorption correction is to obtain the spectral distribution at the chloroplast level (corrected spectrum, $\boldsymbol{I}_{f}^{c}(\lambda)$ ) from the experimental spectrum at leaf level (equation 28). This is generally achieved by multiplying the experimental leaf-level fluorescence spectrum $\boldsymbol{I}_{f}^{e}(\lambda)$ by a correction factor $\boldsymbol{f}$ (see equation 28) that will have different expressions depending on the model used for its deduction.

$I_{f}^{c}(\lambda)=I_{f}^{e}(\lambda) \cdot f$

In the correction methodology proposed by Agati et al. [38], the correction factor $\boldsymbol{f}$ is a function of the reflectance (R) and transmittance $(\boldsymbol{T})$ of a single leaf at the emission and excitation wavelengths ( $\lambda$ and $\lambda_{0}$ respectively) (eq. 29).

$f=\frac{\ln 1 /\left(R_{\lambda_{0}}+T_{\lambda_{0}}\right)+{ }^{\ln 1 /\left(R_{\lambda}+T_{\lambda}\right)}}{\ln 1 /\left(R_{\lambda_{0}}+T_{\lambda_{0}}\right)} \frac{1-R_{\lambda_{0}}-T_{\lambda_{0}}}{1-\left(R_{\lambda_{0}}+T_{\lambda_{0}}\right)\left(R_{\lambda}+T_{\lambda}\right)}$

This approach considered an infinitesimal layer of leaf where fluorescence was generated and was then exponentially attenuated due to light re-absorption. Lateral light loses were neglected and light scattering was taken into account by considering an effective pathlength. This model was validated by comparing the corrected emission spectra for Aurea mutant and for the wild type of tomato leaves, which had different chlorophyll concentration and chloroplast ultrastructure. Matching of both spectra proved the effectiveness of the model.

The second methodology was applied to leaves by Ramos and Lagorio [36] in 2004, but it had already been developed and applied for inert materials in 1998 by Lagorio et al. [40]. It is a two-flux model based on the Kubelka- Munk theory [2]. It assumes that leaves act as ideal diffusers and that the emission produced in each element may be decomposed in two flows with opposite directions and the same magnitude. In this case, the correction factor $f$ is given by eq. 30 .

$f=\left[\frac{1}{1+\sqrt{\frac{F\left(R_{\lambda}\right)+2}{F\left(R_{\lambda}\right)+2}}} \frac{1}{1+\sqrt{\frac{F\left(R_{\lambda}\right)\left[F\left(R_{\lambda}\right)+2\right]}{F\left(R_{\lambda_{0}}\right)\left[F\left(R_{\lambda_{0}}\right)+2\right]}}}\right]^{-1}$

For the derivation of the correction factor according to Lagorio et al. [40], it was assumed that no light was transmitted through the system, so this approach is strictly applicable to opaque samples. Thus, when working with plants, a nontransmitting pile of leaves should be used in fluorescence measurements for the correct application of the approach (Figure 9).

The model was validated by comparing the corrected spectral distribution of fluorescence of a group of stacked leaves with the spectral distribution of fluorescence emerging from a thin layer of chloroplasts. 


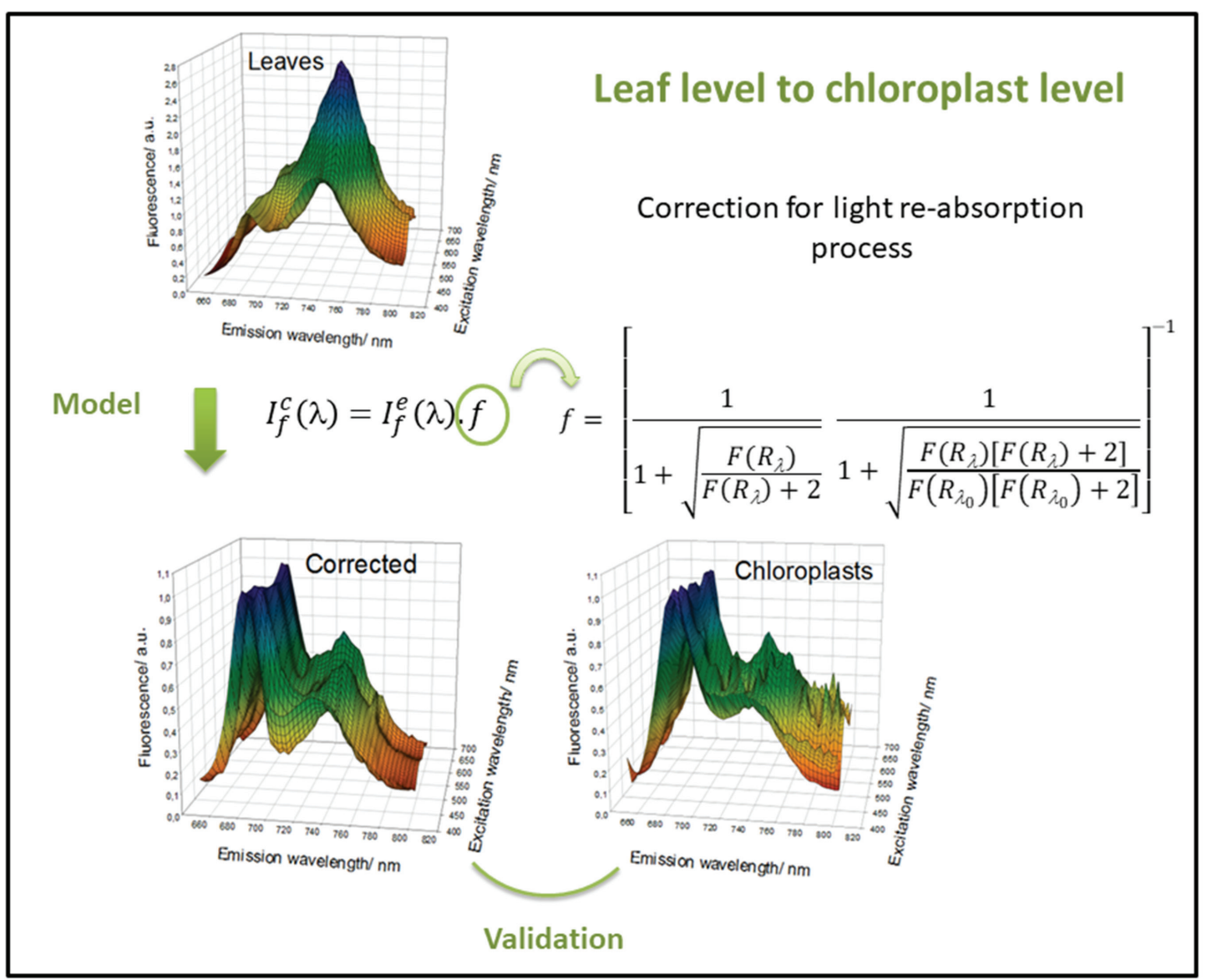

Figure 9. Normalized fluorescence spectra of Ficus benjamina leaves before and after correction for light re-absorption processes. The corrected spectra were compared with those obtained from a thin layer of chloroplasts (free from reabsorption). The corrected spectra agree with chloroplasts spectra thus validating the model. Adapted from [36] with permission from the European Society for Photobiology, the European Photochemistry Association, and The Royal Society of Chemistry.

In principle, a two-flux model is expected to be better than a single flux model. However, in practice, both Agati et al. and Lagorio et al. models give close results. The choice of which one to use will depend in part on the possibility of having only a single leaf or a group of leaves for the measurements.

Leaf fluorescence measurements are carried out by means of a standard spectrofluorometer in front face geometry. In case the method described by Agati et al. is applied for corrections, the experimental fluorescence spectrum should be obtained for a single leaf. On the other side, if the method described by Lagorio et al. is used, it should be recorded for a group of stacked leaves that do not transmit light.

\subsubsection{From the canopy to the leaf}

Remote sensing of chlorophyll fluorescence tracks the emission from a canopy and is becoming an interesting tool for the assessment of plant health from a distance [41]. This technique is currently highly appreciated because it allows the study of anthropogenic or natural effects on large areas of plantations, the adequate monitoring of forests health and crop production and also the estimation of carbon flows between vegetation and the atmosphere which is closely related to global climate change [42].

There are passive and active methods for the remote sensing of chlorophyll fluorescence. Passive methods measure fluorescence excited directly by sunlight as irradiation source [43], whereas active methods use illumination with artificial light, usually high-energy LASERS or LEDS [44]. 
Chlorophyll fluorescence emitted by a canopy is only 1 or $2 \%$ of the light absorbed and is very small compared to the fraction of light reflected. The measuring instrument (spectro-radiometer) used in the field, collects the total radiance composed by both the light reflected by vegetation and a contribution of its fluorescence at each wavelength. In passive methods, the two signals need to be decoupled using the Fraunhofer line discrimination principle (for detailed information see [45]). A very recent work proposed a new and promising active methodology to measure the chlorophyll fluorescence emission spectral distribution of canopies in situ, upon inducing the fluorescence with light emitting diodes at night [44].

Within a canopy, light re-absorption processes are also present and the spectrum recorded for a canopy is distorted from that of a single leaf. It is therefore worthwhile exploring models to correct this distortion. Recently, Romero et al. developed an approach to obtain the spectral distribution of fluorescence at leaf level from the experimental fluorescence spectrum at canopy level [46].

The model assumes that the system is the set composed of canopy plus soil. The vegetation homogenously covers all the land and there is no fraction of bare soil. No light is transmitted through the system and the canopy is formed by units (leaves) that emit fluorescence. Mono exponential attenuation of light within the canopy is assumed for both the excitation light and for the generated fluorescence (which is reduced by the process of re-absorption). Light scattering is taken into consideration by determining an effective pathlength. The excitation beam is considered monochromatic; therefore, this model is applicable to data remotely obtained with active methods. It is not suitable for data produced by passive measuring methods that employ polychromatic light.

According to this approach, to perform the correction, the experimental spectrum should be multiplied by the correction factor which is given by eq. 31 :

$$
f=\left[1+\frac{\ln \xi(\lambda)}{\ln \xi\left(\lambda_{0}\right)}\right]\left[\frac{1-\xi\left(\lambda_{0}\right)}{1-\xi\left(\lambda_{0}\right) \xi(\lambda)}\right]
$$

With

$\xi(\lambda)=\mathrm{R}_{\lambda}+\mathrm{Tc}_{\lambda}\left(1-\mathrm{Rb}_{\lambda}\right)$

$\boldsymbol{R}$, is the total reflectance of the system, $\boldsymbol{T} \boldsymbol{c}_{\lambda}$ is the canopy transmittance and $\boldsymbol{R} \boldsymbol{b}_{\lambda}$ is the soil reflectance at wavelength $l$ in all the cases.

This approach was successfully validated by comparing the corrected spectral distribution of fluorescence from the canopy with that experimentally obtained for a leaf (Figure 10).

Thus, by the successive application of the models described above "canopy to leaf" and "leaf to chloroplast" (sections 2.4.1.2. and 2.4.1.1), it is possible to retrieve the spectral distribution of fluorescence at chloroplast level from that measured at canopy level. This is the fundamental feature of this modelling, which permits to derive information at microscopic level from data recorded at a scale about one million times larger. 


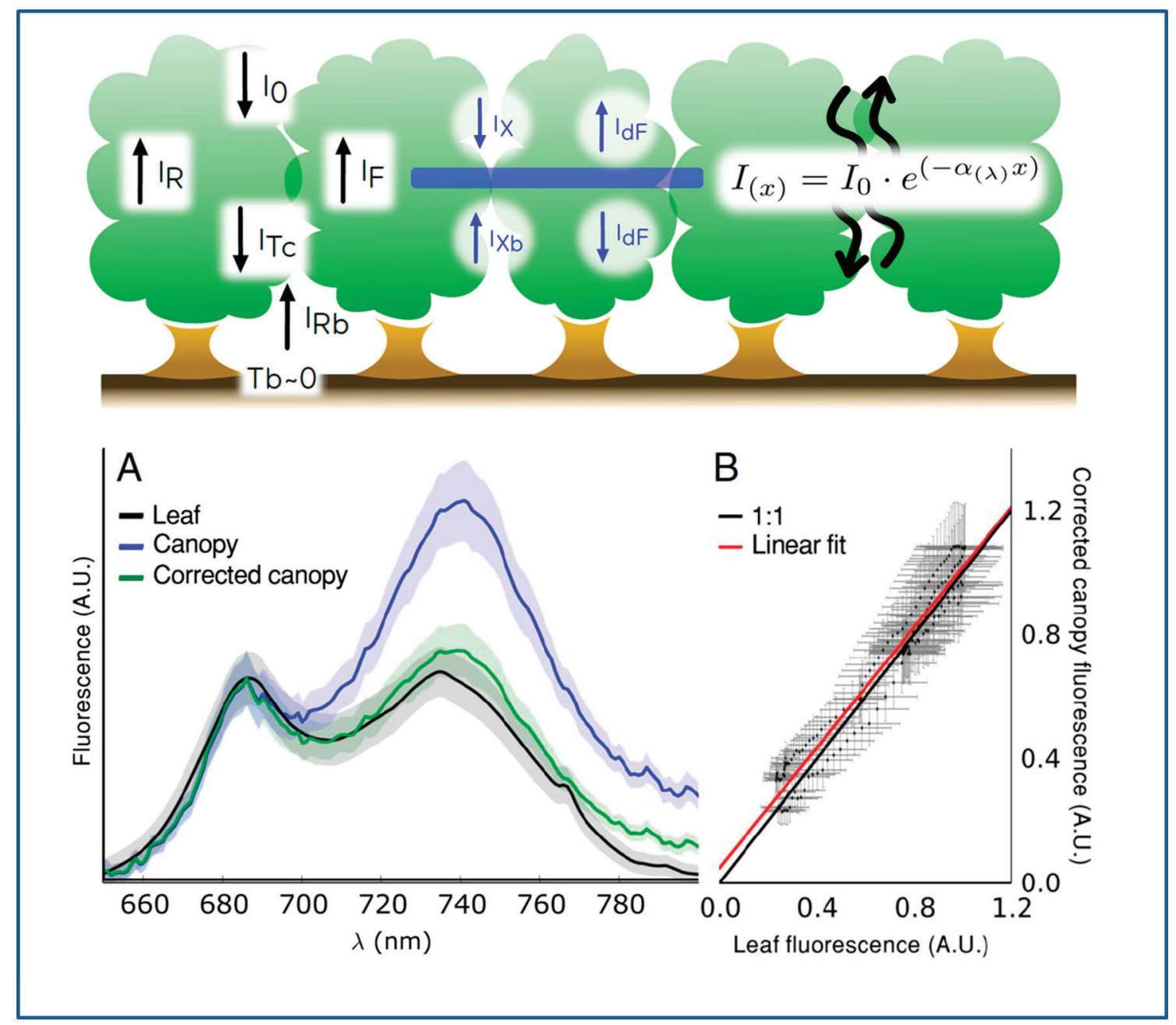

Figure 10. Fluorescence spectra and model validation: from canopy to leaf level. (A) Normalized fluorescence spectra for leaf (black), canopy (blue) and corrected canopy (green). Shadows show the standard error. (B) Correlation between the canopy fluorescence corrected by light re-absorption and leaf fluorescence. Linear fit: $\mathrm{y}=(0.97 \pm 0.02) * \mathrm{x}+(0.05 \pm$ 0.01), $\left.\mathrm{R}^{2}=0.94\right)$. Adapted with permission from [46], Copyright 2018 Elsevier.

\section{Reflectance and Fluorescence. Practical applications}

The analysis of the light reflected or emitted as fluorescence by organisms is an excellent tool for the development of non-destructive methods for quality and health assessment. These methods are attractive for monitoring the status of countless products. Some cases of application in foodstuff, in crops production and in environmental issues developed in our research group, are illustrated below.

Biospectroscopy and imaging were used to evaluate the quality of yerba mate (YM), a commercial product composed of dried leaves of Ilex paraguariensis mixed with fragments of dried sticks. This herb is extensively used in South America to prepare drinkable infusions (Figure 11) rich in polyphenols and antioxidants [47]. The results were complemented with bio-spectrometric studies based on MALDI techniques [48]. The stick/leaf mass ratio is a standard parameter in the quality control of yerba mate, which is limited by food code regulations. The nutraceutical content is higher in leaves than in stems and therefore the frequent adulteration of yerba mate with the addition of powdered stick leads to a reduction in the quality of the product. In effect, from MALDI analysis, it was shown that rutin and metasaponins 2 and 4, which have healing properties, are found in leaves and missing in sticks (both for the intact product and for the infusions) [47]. The excitation-emission matrixes of YM excited in the UV (300-400 nm) showed emission from YM in the blue and in the red part of electromagnetic spectrum. The fluorescence ratio Blue $(469 \mathrm{~nm}) / \operatorname{Red}(680 \mathrm{~nm})$ displayed a linear relation with the percentage of sticks in the sample. Additional linear correlations were found between other optical parameters as sample reflectance at $669 \mathrm{~nm}$ or color coordinates (RGB) and the sample composition in percentage of sticks [47]. These results offer the chance to use non-destructive methods to determine the yerba-mate composition. Moreover, as fluorescence and reflectance differ for the stick and the leaf, it is possible to detect adulteration with stick, when it has been added in ground form. 


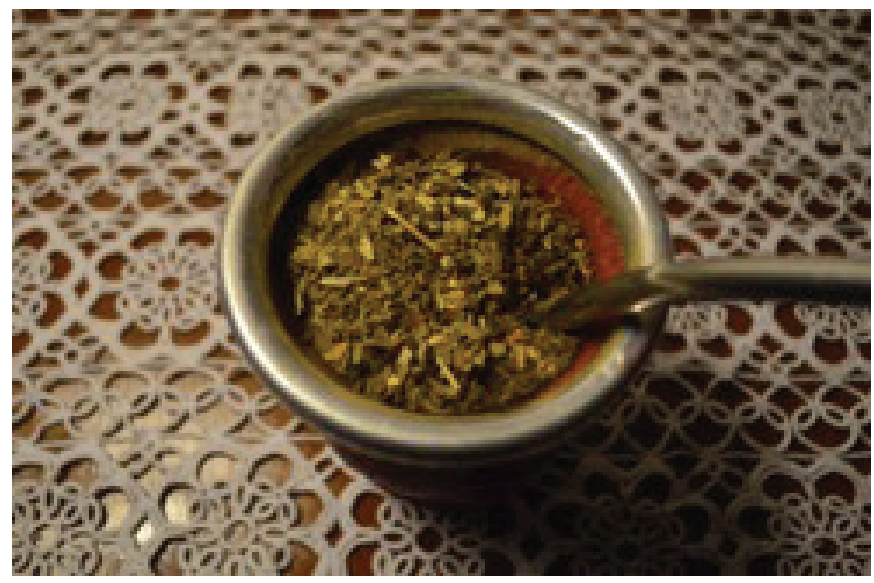

Figure 11. Yerba Mate is extensively used in South America to prepare drinkable infusions (Mate) rich in polyphenols and antioxidants.

A similar initiative was carried out for oregano (Origanum vulgare), which is an herb with properties used in medicine, cosmetics, flavors and scents and which is marketed as a mixture of dried leaves and inflorescences with a maximum of $3 \%$ of stems. This percentage is particularly important for its essential oil content, which is rich in phenolic monoterpenes and carvacrol [49]. The essential oil is contained in trichomes and the density of these glands follows the order: inflorescences $>$ leaves $>$ stems (Figure 12). The various organs of the oregano plant showed fluorescence emission in the blue, green, red and far red part of the electromagnetic spectrum. For leaves, the ratio of fluorescence intensities green to far-red and blue to far-red maintained a linear relationship with water content. In addition, at constant humidity the blue/far-red peak ratio was a fingerprint for each organ of the plant. It was further observed that, for the commercial product, both the fluorescent emission at $310 \mathrm{~nm}$ (induced by excitation at $275 \mathrm{~nm}$ ) and the absorption band at $811 \mathrm{~cm}^{-1}$ obtained by attenuated total reflectance Fourier transform infrared spectroscopy could be correlated with the essential oil content (details can be found in [49]).

The non-destructive determination of moisture in intact packaged oregano by measuring the diffuse reflectance in the near IR (through the packaging) has also been reported. This methodology is applicable as long as the wrapping film does not show absorption at 1440 or $1920 \mathrm{~nm}$ [50]. 

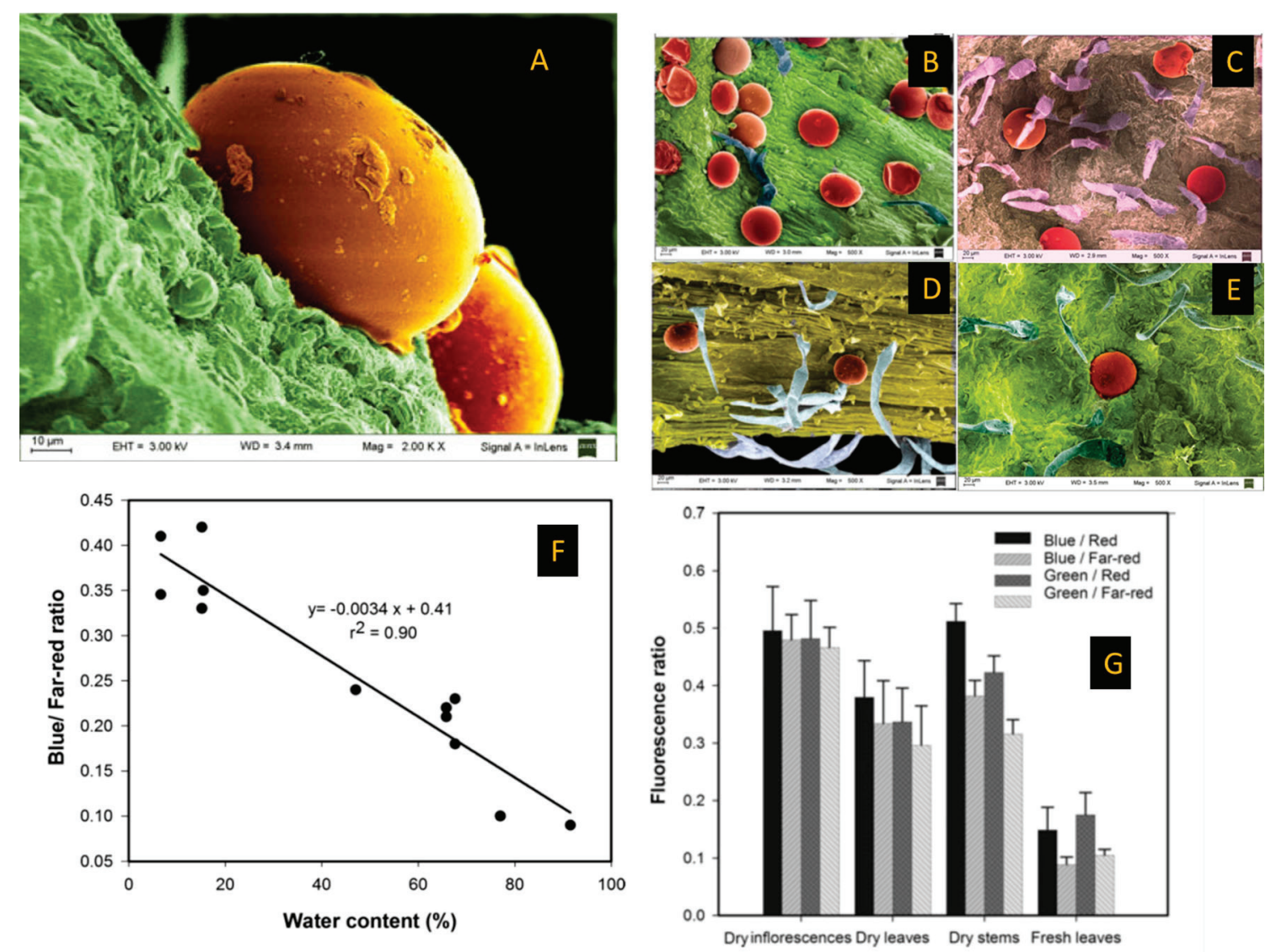

Figure 12. Oregano. A) SEM image in false colours for oregano glandular trichomas containing the essential oil (2000 $\mathrm{x}), \mathrm{B}), \mathrm{C}), \mathrm{D})$ and E) SEM images in false colours $(500 \mathrm{x}$ ) for dry floral bracts, dry flower, dry stem and dry leaf respectively, F) Linear correlation between the fluorescence ratio Blue/Far-red and the water content in oregano leaves, G) Fluorescence ratios for oregano organs. Adapted with permission from [49] Copyright 2013 Wiley.

The analysis of variable chlorophyll fluorescence, described in section 3.4, was successfully applied in literature for the post-harvest screening of fruit and vegetables [5]. Recently, this technique was used to monitor the status of kiwifruit [22] and eggplant [23] under different storage conditions.

Our research group also used reflectance and chlorophyll fluorescence for environmental applications such as the study of the effect of diverse pollutants on plants: arsenic [51-53], combined presence of arsenic, vanadium, boron and fluoride [54], atrazine and methyl viologen [27] and nanoparticles [55], among others.

In addition, works related to agronomic sciences were carried out. Reflectance and chlorophyll fluorescence were used to detect phosphorous deficiency in crops [56]. Reflectance and radiance measurements were also recorded at canopy level in grass species during their senescence. The sun induced-chlorophyll fluorescence was extracted from the radiance spectra of canopies using the Fraunhofer Line Discrimination-method and was correlated with reflectance indexes used in literature as proxy for estimation of the Radiation Use Efficiency (RUE) [57].

\section{Conclusions}

The study of the interaction between light and biological entities is fundamental both to understand basic functions of the entities as well as for the development of new non-destructive technologies of analysis. As shown in this review, physical modelling greatly assists in the interpretation of the phenomena that take place upon illumination. For improved practical applications in the near future, the knowledge arising in this area should be integrated with other fields of science such as machine learning, remote sensing, artificial intelligence and computational vision. It is hoped that the new intelligent photonic technologies will be implemented in drones, robots and photonic sensor imaging. 
Considering the transdisciplinary nature of this kind of projects, future success will surely rely on the degree of interaction among different sciences such as Physics, Chemistry, Biology, Bioengineering, Electronics and Computer Science.

\section{Acknowledgments}

The authors are grateful to the University of Buenos Aires (UBACyT 20020130100166BA, 20020170100037BA and 20020170200118BA) and to the Agencia Nacional de Promoción Científica y Tecnológica (PICT 2012-566 2357, PICT 2015-0396 and PICT 2015-820) for the financial support. JR developed his work with a fellowship from CONICET. MGL, AI, GBC and JF are Researchers of CONICET (Argentina). CT was supported by a Human Frontier Science Program (LT 000660/2018-L) postdoctoral fellowship. We also thank M. E. Ramos, J. Mendes Novo, B. Ospina Calvo, T.L. Parapugna, G. Petroselli, R. Erra, S. Gismondi, A. Nievas, V. Diz, R. Torres, C. Marchi, J. Paruelo, A. Barreira, P. Tubaro, G. Dundas, A. Fernández Cirelli for their collaboration in works related to the topic of this review. This review is dedicated to the memory of Professor Enrique San Román who introduced us in the study of high light- scattering materials.

\section{References}

[1] Jurgens, M.; Mayerhöfer, T.; Popp, J. Introduction to Biophotonics. In Handbook of Biophotonics. Vol.1: Basics and Techniques, First Edition. Popp, J; Tuchin, V. V.; Chiou, A; and Heinemann, S. H., Ed.; Wiley-VCH Verlag GmbH \& Co, 2011, Vol.1; ch. 1, p.1.

[2] Rodríguez, H. B.; Mirenda, M.; Lagorio, M. G.; San Román, E. Photophysics at Unusually High Dye Concentrations. Acc. Chem. Res., 2019, 52, 1, 110-118.

[3] Biophotonics for Medical Applications. In Woodhead Publishing Series in Biomaterials Book; Meglinski, I., Ed.; Elsevier Ltd., 2015.

[4] Martinelli, F., Scalenghe, R., Davino, S., Panno, S., Scuderi, G., Ruisi, P., Villa, P., Stroppiana, D., Boschetti, M., Goulart, L. R., Davis, C. E., Dandekar, A. M. Advanced methods of plant disease detection. A review. Agron. Sustain. Dev., 2015, 35, 1, 1-25.

[5] DeEll, J. R.; Toivonen, P. M. A. Use of chlorophyll fluorescence in postharvest quality assessments of fruits and vegetables. In Practical applications of Chlorophyll fluorescence in plant biology. DeEll, J. R.; Toivonen, P. M. A., Ed. Kluwer Academic Publishers, London, 2003, ch. 7, p. 203.

${ }^{[6]}$ Iriel, A.; Lagorio, M. G. Implications of reflectance and fluorescence of Rhododendron indicum flowers in biosignaling. Photochem. Photobiol. Sci., 2010, 9, 342-348.

[7] Wendlandt, W. W.; Hecht, H. G., Reflectance Spectroscopy, Wiley, New York, 1966.

[8] Cordon, G. B.; Lagorio, M. G. Optical properties of the adaxial and abaxial faces of leaves. Chlorophyll fluorescence, absorption and scattering coefficients. Photochem. Photobiol. Sci., 2007, 6, 873-882.

[9] Iriel, A. and Lagorio M. G. Biospectroscopy of Rhododendron indicum flowers. Non-destructive assessment of anthocyanins in petals using a reflectance-based method. Photochem. Photobiol. Sci., 2009, 8, 337-344.

${ }^{[10]}$ Cordon, G. B.; Gismondi, S.; Nievas, A. V.; Lagorio, M. G.: Non-destructive assessment of water and pigments in leaves from the remission function using the Kubelka-Munk theory. Proceedings of the International Color Association, 2010, 397-400. ISSN 0280-2198.

${ }^{[11]}$ http://www.brucelindbloom.com/

${ }^{[12]}$ Cordon, G. B.; Lagorio, M. G. Sensing chlorophyll, carotenoids and anthocyanin concentration in leaves with spatial resolution from digital image. In Color in Food: Technological and Psychophysical Aspects. CRC Press, New York, USA. ISBN 978-1-43987-693-0, 2012, Ch.13, 121-132.

${ }^{[13]}$ Leyre, S.; Coutino-Gonzalez, E; Joos, J. J.; Ryckaert, J.; Meuret, Y.; Poelman, D.; Smet, P. F.; Durinck, G.; Hofkens, J.; Deconinck, G.; Hanselaer, P. Absolute determination of photoluminescence quantum efficiency using an integrating sphere setup. Rev. Sci. Instrum., 2014, 85, 123115. 
${ }^{[14]}$ Valenta, J. Determination of absolute quantum yields of luminescing nanomaterials over a broad spectral range: from the integrating sphere theory to the correct methodology. Nanosci. Meth., 2014, 3, 11-27.

[15] Würth, C.; Resch-Genger, U. Determination of Photoluminescence Quantum Yields of Scattering Media with an Integrating Sphere: Direct and Indirect Illumination. Appl. Spectrosc., 2015, 69, 6, 749-759.

${ }^{[16]}$ Lagorio, M. G. Tutorial: Determination of Fluorescence Quantum Yields in Scattering Media. Methods Appl. Fluoresc., $2020,8,4,043001$.

${ }^{[17]}$ Wrighton, M. S; Ginley, D. S.; Morse, D. L. A Technique for the Determination of Absolute Emission Quantum Yields of Powdered Samples. J. Phys. Chem., 1974, 78, 2229-2233.

${ }^{[18]}$ Iriel, A.; Lagorio, M. G. Is the flower fluorescence relevant in biocommunication? Naturwissenschaften, 2010, 97, 10, 915-924.

${ }^{[19]}$ Barreira, A. S.; Lagorio, M. G.; Lijtmaer, D. A.; Lougheed, C.; Tubaro, P. L. Fluorescent and ultraviolet sexual dichromatism in the blue-winged parrotlet. J. Zoology, 2012, 288, 135-142.

${ }^{[20]}$ Taboada, C.; Brunetti, A. E.; Pedron,F. N.; Carnevale Neto, F.; Estrin, D. A.; Bari, S. E.; Chemes, L. B.; Peporine Lopes, N.; Lagorio, M. G.; Faivovich, J. Naturally occurring fluorescence in frogs. PNAS, 2017, 114, $3672-3677$.

${ }^{[21]}$ Lagorio, M. G.; Cordon, G. B.; Iriel, A. Reviewing the relevance of fluorescence in biological Systems. Photochem. Photobiol. Sci., 2015, 14, 1538-1559.

${ }^{[22]}$ Mendes Novo, J.; Iriel, A.; Lagorio, M. G. Modelling chlorophyll fluorescence of kiwi fruit (Actinidia deliciosa), Photochem. Photobiol. Sci., 2012, 11, 724-730.

${ }^{[23]}$ Ospina Calvo, B.; Parapugna, T. L.; Lagorio, M. G. Variability in chlorophyll fluorescence spectra of eggplant fruit grown under different light environments: a case study. Photochem. Photobiol. Sci., 2017, 16, 711-710.

${ }^{[24]}$ Lang, M.; Stober, F.; Lichtenthaler, H. K. Fluorescence emission spectra of plant leaves and plant constituents. Radiat. Environ. Biophys., 1991, 20, 333-347.

${ }^{[25]}$ Pfündel, E. Estimating the contribution of photosystem I to total leaf chlorophyll fluorescence. Photosynth. Res., 1998, $56,2,185-195$.

${ }^{[26]}$ Lichtenthaler, H. K.; Buschmann, C.; Knapp, M. How to correctly determine the different chlorophyll fluorescence parameters and the chlorophyll fluorescence decrease ratio RFd of leaves with the PAM fluorometer. Photosynthetica, $2005,43,379-393$.

${ }^{[27]}$ Iriel, A.; Mendes Novo, J.; Cordon, G. B.; Lagorio, M. G., 2014. Atrazine and methyl viologen effects on chlorophyll-a fluorescence revisited. Implications in photosystems emission and ecotoxicity. Photochem. Photobiol., 2014, 90, 107112.

${ }^{[28]}$ Kornyeyev D.; Hendrickson L. Research note: Energy partitioning in photosystem II complexes subjected to photoinhibitory treatment. Funct Plant Biol., 2007, 34, 3, 214-220.

${ }^{[29]}$ Genty B.; Briantais J-M.; Baker N. R. The relationship between the quantum yield of photosynthetic electron transport and quenching of chlorophyll fluorescence. Biochim. Biophys. Acta (BBA) - General Subj., 1989, 990, 1, 87-92.

${ }^{[30]}$ Hendrickson L.; Furbank R.T.; Chow W. S. A simple alternative approach to assessing the fate of absorbed light energy using chlorophyll fluorescence. Photosynth. Res., 2004, 82, 1, 73-81.

${ }^{[31]}$ Guadagno, C. R.; Virzo De Santo, A.; D’Ambrosio, N. A revised energy partitioning approach to assess the yields of non-photochemical quenching components. Biochim Biophys Acta - Bioenerg., 2010, 1797, 5, 525-530.

${ }^{[32]}$ Hendrickson, L.; Förster, B.; Pogson, B. J.; Wah, S. C. A simple chlorophyll fluorescence parameter that correlates with the rate coefficient of photoinactivation of photosystem II. Photosynth. Res., 2005, 84, 43-49.

[33] Ospina Calvo, B.; Lagorio, M. G. Quantitative Effects of Pigmentation on the Re-absorption of Chlorophyll a Fluorescence and Energy Partitioning in leaves. Photochem Photobiol., 2019, 95, 6, 1360-1368.

${ }^{[34]}$ Franck, F.; Juneau, P.; Popovic, R. Resolution of the photosystem I and photosystem II contributions to chlorophyll fluorescence of intact leaves at room temperature. Biochim. Biophys. Acta, 2002, 1556, 2, 239-246. 


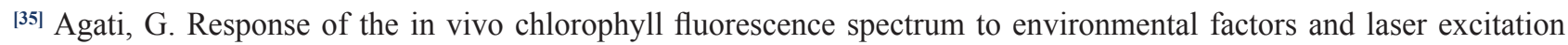
wavelength. Pure Appl. Opt. 1998, 7, 4, 797-807.

${ }^{[36]}$ Ramos, M. E.; Lagorio, M. G. True fluorescence spectra of leaves. Photochem. Photobiol. Sci., 2004, 3, 1063-1066

${ }^{[37]}$ Cordon, G. B.; Lagorio, M. G. Re-absorption of chlorophyll fluorescence in leaves revisited. A comparison of correction models. Photochem. Photobiol. Sci., 2006, 5, 735-740.

${ }^{[38]}$ Agati, G; Fusi, F.; Mazzinghi, P.; Lipucci di Paola, M. A simple approach to the evaluation of the re-absorption of chlorophyll fluorescence spectra in intact leaves. J. Photochem. Photobiol., B, 1993, 17, 163-171.

${ }^{[39]}$ Gitelson, A. A.; Buschmann, C.; Lichtenthaler, H. K. Leaf Chlorophyll Fluorescence corrected for re-absorption by means of absorption and reflectance measurements. J. Plant Physiol., 1998, 152, 283-296.

${ }^{[40]}$ Lagorio, M. G.; Dicelio, L. E.; Litter, M. I.; San Román, E. Modeling of Fluorescence Quantum Yields of Supported dyes. Aluminum carboxyphthalocyanine on cellulose. J. Chem. Soc., Faraday Trans., 1998, 94, 3, 419-425.

${ }^{[41]}$ Guanter, L.; Zhang, Y.; Jung, M.: Joiner, J.; Voigt, M.; Berry, J. A.; Frankenberg, C.; Huetef, A. R.; Zarco-Tejada, P. J.; Lee, J. -E.; Moran, M. S.; Ponce-Campos, G.; Beer, C.; Camps-Valls, G.; Buchmann, N.; Gianelle, D.; Klumpp, K.; Cescatti, A.; Baker, J. M.; Griffis, T. J. Global and time-resolved monitoring of crop photosynthesis with chlorophyll fluorescence. Proc. Natl. Acad. Sci. U. S. A., 2014, 111, 14, E1327-E1333.

${ }^{[42]}$ Grace, J.; Nichol, C.; Disney, M.; Lewis, P.; Quaife, T.; Bowyer, P., Can we measure terrestrial photosynthesis from space directly, using spectral reflectance and fluorescence? Glob. Ch. Biol., 2007, 13, 7, 1484-1497.

${ }^{[43]}$ Meroni, M.; Rossini, M.; Guanter, L.; Alonso, L.; Rascher, U.; Colombo, R.; Moreno, J. Remote sensing of solarinduced chlorophyll fluorescence: Review of methods and applications. Remote Sens. Environ., 2009, 113, 10, 2037 -2051 .

${ }^{[44]}$ Atherton, J.; Liu, W.; Porcar-Castell, A. Nocturnal Light Emitting Diode Induced Fluorescence (LEDIF): A new technique to measure the chlorophyll a fluorescence emission spectral distribution of plant canopies in situ. Remote Sens. Environ., 2019, 231, 111137.

${ }^{[45]}$ Alonso, L.; Gómez-Chova, L.; Vila-Francés, J.; Amorós-López, J.; Guanter, L.; Calpe, J.; Moreno, J. Improved Fraunhofer Line Discrimination Method for Vegetation Fluorescence Quantification. IEEE Geosci. Remote S., 2008, $5,4,620-624$

${ }^{[46]}$ Romero, J. M.; Cordon, G. B.; Lagorio, M. G. Modeling re-absorption of fluorescence from the leaf to the canopy level. Remote Sens. Environ., 2018, 204, 138-146.

${ }^{[47]}$ Parapugna, T. L.; Petroselli, G.; Erra-Basells, R.; Lagorio, M. G. Biospectroscopy, biospectrometry and imaging of Ilex paraguariensis. Basis for non-destructive quality evaluation using artificial vision. Photochem. Photobiol. Sci, $2016,15,879-888$

${ }^{[48]}$ Petroselli, G.; Parapugna, T. L.; Lagorio, M. G.; Erra-Basells, R. MALDI- and LDI-MS saponin fingerprint of leaves and sticks components of commercial yerba mate (Ilex paraguariensis). J. Mass Spectrom., 2019, 54, 2, $195-203$.

${ }^{[49]}$ Mendes Novo, J.; Iriel, A.; Marchi, M. C.; Lagorio, M. G.. Spectroscopy, Microscopy and Fluorescence Imaging of Origanum vulgare L. Basis for Non-destructive Quality Assessment. Photochem. Photobiol. 2013, 89, 1383-1390.

${ }^{[50]}$ Mendes Novo, J.; Iriel, A.; Lagorio, M. G. Rapid spectroscopic method to assess moisture content in free and packaged oregano (Origanum vulgare L.) JARMAP, 2016, 3, 211-214.

${ }^{[51]}$ Iriel, A.; Dundas, G.; Fernández Cirelli, A.; Lagorio, M. G. Effect of arsenic on reflectance spectra and chlorophyll fluorescence of aquatic plants. Chemosphere, 2015, 119, 697-703.

${ }^{[52]}$ Iriel, A.; Lagorio, M. G.; Fernández Cirelli, A. Biosorption of arsenic from groundwater using Vallisneria gigantea plants. Kinetics, equilibrium and photophysical considerations. Chemosphere, 2015, 138, 383-389.

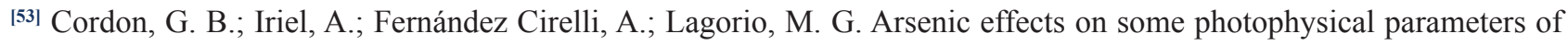
Cichorium intybus under different radiation and water irrigation regimes. Chemosphere 2018, 204, 398-404.

${ }^{[54]}$ Iriel, A.; Cordon, G. B.; Fernández Cirelli, A.; Lagorio, M. G. Non-destructive methodologies applied to track the 
occurrence of natural micropollutants in watering: Glycine max as a biomonitor. Ecotoxicol. Environ. Saf., 2019, 182, 109368.

${ }^{[55]}$ Torres, R.; Diz, V.; Lagorio, M. G. Effects of gold nanoparticles on the photophysical and photosynthetic parameters of leaves and chloroplasts. Photochem. Photobiol. Sci., 2018, 17, 505-516.

${ }^{[56]}$ Yaryura, P., Cordon, G., Leon, M., Kerber, N., Pucheu, N., Rubio, G., García, A., Lagorio, M. G., Effect of phosphorus deficiency on reflectance and chlorophyll fluorescence of cotyledons of oilseed rape (Brassica napus L.). J. Agron. Crop Sci., 2009, 195, 186-196.

${ }^{[57]}$ Cordon, G. B.; Lagorio, M. G.; Paruelo, J. Chlorophyll fluorescence, photochemical reflective index and normalized difference vegetative index during plant senescence. J. Plant Physiol., 2016, 199, 100-110.

\section{Bios}

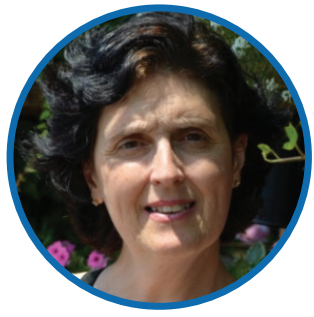

María Gabriela Lagorio

María Gabriela Lagorio was born in Buenos Aires, Argentina. She obtained her B.S. (Chemical Sciences) in 1982 at UBA. She worked in the private industry from 1983 to 1987. Subsequently, she started her research activities, obtaining her $\mathrm{PhD}$ in Chemical Sciences (UBA) in 1991. She is presently Professor at UBA and scientist researcher of CONICET. She is the head of the Research Group in Photochemistry and Photobiology in the Department of Inorganic, Analytical and Physical Chemistry/ INQUIMAE (FCEN, UBA). Her research field is focused on the modeling and analysis of the interaction of light with biological entities.

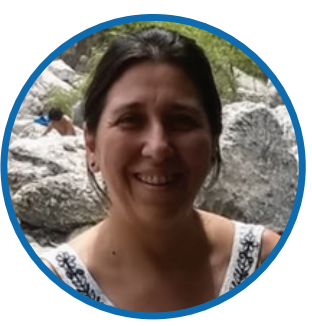

\section{Gabriela B. Cordon}

Gabriela B. Cordon is Associate Scientist researcher of the National Research Council of Argentina (CONICET) and Assistant Professor at Faculty of Agronomy of Universidad de Buenos Aires. She is member of the Regional analysis and remote sensing laboratory (LART) and of the Agricultural plant physiology and ecology research institute (IFEVA). Her research focuses on the use of chlorophyll fluorescence and reflectance spectroscopy as non-destructive tools for agricultural and environmental monitoring at the leaf, plant and canopy level.

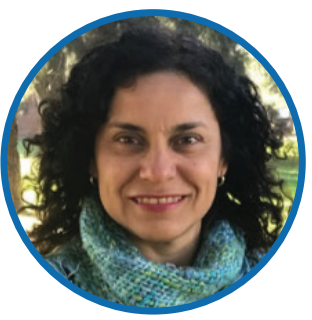

\section{Analia Iriel}

Analia Iriel was born in Santa Fe, Argentina in 1975. She received her chemistry degree in 1999 from the University of the Litoral. In 2006 she obtained her $\mathrm{PhD}$ at the University of Buenos Aires under the supervision of Enrique San Román. She is currently Associate Professor at the Science and Technology School of San Martin University. She is an Associate Scientist researcher of CONICET. Her research interests include micropollutants in environmental matrixes, phytoremediation technologies to remove arsenic from groundwater and non-destructive methodologies to assess environmental risk associated to the presence of pesticides from agricultural activities.

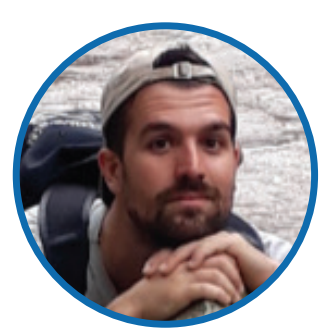

Juan Manuel Romero

Juan Manuel Romero was born in Buenos Aires, Argentina. He has a degree in Biology (FCEN, UBA). Currently, he is completing his doctoral thesis under the direction of M. G. Lagorio and G. Cordon, in modeling the interaction of light with photosynthetic material, with the aim of using fluorescence as an indicator of physiological state. He is a CONICET fellow and a teacher in the Department of Inorganic, Analytical and Physical Chemistry.

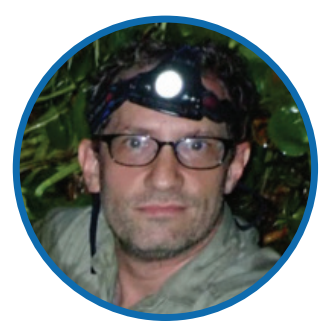

\section{Julián Faivovich}

Julián Faivovich finished his undergraduate studies in biology in the Facultad de Ciencias Exactas y Naturales, Universidad de Buenos Aires, Buenos Aires, Argentina in 1999, and his doctoral studies in 2005, in Columbia University and the American Museum of Natural History, New York, U.S.A. After two years as a postdoc student in the Universidade Estadual Paulista, Rio Claro, São Paulo, Brazil, he returned to Argentina in 2008 as a researcher in CONICET, in charge of the herpetology division of the Museo Argentino de Ciencias Naturales "Bernardino Rivadavia." His research has always been focused in systematics and biology of amphibians. 


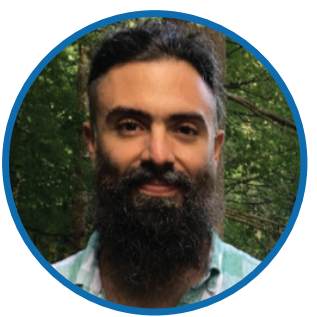

\section{Carlos Taboada}

Carlos Taboada received his $\mathrm{PhD}$ in Biology in 2017 from the University of Buenos Aires. He is currently a Human Frontier Science Program Fellow at Duke University, USA. His research focuses on organismal biology and combines various theoretical and experimental approaches in photophysics, biochemistry, spectroscopy and mass spectrometry to understand the basis of animal coloration and visual ecology. 